

\title{
The influence of non-uniform ambient noise on crustal tomography in Europe
}

Piero Basini, Tarje Nissen-Meyer, Lapo Boschi, Emanuele Casarotti, Julie Verbeke, Olaf Schenk, Domenico Giardini

\section{- To cite this version:}

Piero Basini, Tarje Nissen-Meyer, Lapo Boschi, Emanuele Casarotti, Julie Verbeke, et al.. The influence of non-uniform ambient noise on crustal tomography in Europe. Geochemistry, Geophysics, Geosystems, 2013, pp.1-52. 10.1002/ggge.20081 . hal-00816943

\section{HAL Id: hal-00816943 \\ https://hal.science/hal-00816943}

Submitted on 23 Apr 2013

HAL is a multi-disciplinary open access archive for the deposit and dissemination of scientific research documents, whether they are published or not. The documents may come from teaching and research institutions in France or abroad, or from public or private research centers.
L'archive ouverte pluridisciplinaire HAL, est destinée au dépôt et à la diffusion de documents scientifiques de niveau recherche, publiés ou non, émanant des établissements d'enseignement et de recherche français ou étrangers, des laboratoires publics ou privés. 


\section{The influence of non-uniform ambient noise on 2 crustal tomography in Europe}
P. Basini ${ }^{1}$,
T. Nissen-Meyer ${ }^{2}$, L. Boschi ${ }^{3}$,
E. Casarotti ${ }^{4}$, J. Verbeke $^{5}$,
O. Schenk ${ }^{6}$ and D. Giardini ${ }^{2}$

P. Basini, Department of Physics, University of Toronto, Toronto, Ontario, Canada M5S 1A7

L. Boschi, ISTEP, UMR 7193, UPMC Universite Paris 6, CNRS, FR-75005 Paris, France

E. Casarotti, INGV, via di Vigna Murata 605, 00143 Roma, Italy

D. Giardini, Institute of Geophysics, ETH Zurich, Sonneggstrasse 5, Zurich, Switzerland.

T. Nissen-Meyer, Institute of Geophysics, ETH Zurich, Sonneggstrasse 5, Zurich, Switzerland.

Olaf Schenk, Institute of Computational Science, University of Lugano, Via Giuseppe Buffi 13, Lugano, Switzerland.

J. Verbeke, Institute of Geophysics, Lamont-Doherty Earth Observatory, Columbia University, USA.

${ }^{1}$ Department of Physics, University of 
3 Abstract. Ambient-noise seismology is of great relevance to high-resolution

${ }_{4}$ crustal imaging, thanks to the unprecedented dense data coverage it affords

${ }_{5}$ in regions of little seismicity. Under the assumption of uniformly distributed

6 noise sources, it has been used to extract the Greens function between two

7 receivers. We determine the imprint of this assumption by means of wave prop-

s agation and adjoint methods in realistic 3D Earth models. In this context,

9 we quantify the sensitivity of ambient-noise cross correlations from central

${ }_{10}$ Europe with respect to noise-source locations and shear wavespeed structure.

$\overline{\text { Toronto, }}$ Toronto, Ontario, Canada M5S

1A7

${ }^{2}$ Institute of Geophysics, ETH Zurich,

Sonneggstrasse 5, Zurich, Switzerland.

${ }^{3}$ ISTEP, UMR 7193, UPMC Universite

Paris 6, CNRS, FR-75005 Paris, France

${ }^{4}$ INGV, via di Vigna Murata 605, 00143

Roma, Italy

${ }^{5}$ Lamont-Doherty Earth Observatory,

Columbia University, USA

${ }^{6}$ Institute of Computational Science,

University of Lugano, Via Giuseppe Buffi

13, Lugano, Switzerland. 
${ }_{11}$ We use ambient noise recorded over one year at 196 stations, resulting in a

${ }_{12}$ database of 864 cross-correlations. Our mesh is built upon a combined crustal

${ }_{13}$ and 3D tomographic model. We simulate synthetic ambient-noise cross-correlations

${ }_{14}$ in different frequency bands using a 3D spectral-element method. Traveltime

${ }_{15}$ cross-correlation measurements in these different frequency bands define the

16 misfit between synthetics and observations as a basis to compute sensitiv-

${ }_{17}$ ity kernels using the adjoint method. We perform a comprehensive analy-

18 sis varying geographic station and noise-source distributions around the Eu-

19 ropean seas. The deterministic sensitivity analysis allows for estimating where

${ }_{20}$ the starting crustal model shows better accordance with our dataset and gain

${ }_{21}$ insight into the distribution of noise sources in the European region. This

${ }_{22}$ highlights the potential importance to consider localized noise distributions

${ }_{23}$ for tomographic imaging and forms the basis of a tomographic inversion in

${ }_{24}$ which the distribution of noise sources may be treated as a free parameter

25 similar to earthquake tomography. 


\section{Introduction}

${ }_{26}$ The European lithosphere is characterized by the presence of many microplates whose ${ }_{27}$ motion is dominated by convergence between Africa and Eurasia [e.g., Schmid et al., 2004;

${ }_{28}$ Boschi et al., 2010]. This results in the formation of strong 3D structural lateral variations ${ }_{29}$ which are difficult to image.

so Nowadays, the majority of regional- and global-scale tomography models is based on ${ }_{31}$ the information obtained by either P- or S-wave traveltimes [Bijwaard and Spakman, 32 2000] or by surface-wave dispersion recorded from teleseismic events [Chang et al., 2010; з3 Boschi et al., 2009; Zhu et al., 2012]. While body waves are only partially sensitive to ${ }_{34}$ the structure of the crustal layers [Schivardi and Morelli, 2009], teleseismic surface waves ${ }_{35}$ are too rapidly attenuated to generate high-quality measurements at periods below $30 \mathrm{~s}$ ${ }_{36}$ [Verbeke et al., 2012]. Resolution is further hampered by the strong non-uniformity in the ${ }_{37}$ source-receiver distribution [e.g. Schaefer et al., 2011].

38 Ambient noise interferometry, applied in several different disciplines [Aki, 1957; Cox, 39 1973; Duvall et al., 1993; Shapiro et al., 2005], has been used to retrieve signals reminis40 cent of Green's functions between two receivers from the diffuse wavefield that receivers ${ }_{41}$ continuously record in the absence of earthquakes. It then becomes possible to compile 42 large high-quality surface-wave databases and to perform surface wave tomography [e.g. ${ }_{43}$ Shapiro and Campillo, 2004; Sabra et al., 2005; Verbeke et al., 2012], wherever a dense ${ }_{44}$ station coverage exists.

${ }_{45}$ Usually, seismic ambient-noise in the period-range 8-30 s considered in this work, shows ${ }_{46}$ two maxima in its spectrum between 10 and $20 \mathrm{~s}$ and between 5 and $10 \mathrm{~s}$ [e.g. Stehly et al., 
${ }_{47}$ 2006; Yang et al., 2008]. Hasselmann [1963] relates the first maximum to the interaction of

${ }_{48}$ ocean swells with the shallow sea floor, while Longuet-Higgins [1950] identify the origin of

${ }_{49}$ the second one as the nonlinear interaction between ocean waves propagating in opposite

${ }_{50}$ directions.

${ }^{51}$ The basic idea of ambient-noise seismology is the following: a cross-correlation recorded ${ }_{52}$ at two seismic stations (herafter correlogram as in [Tanimoto, 2008]) is produced by the

${ }_{53}$ interaction of waves equipartitioned in direction with random phases, and contains coher-

${ }_{54}$ ent signals that travel between the two stations. An often-made assumption postulates a

${ }_{55}$ uniform distribution of ambient noise sources surrounding the stations. This is however

56 not valid in most regional-scale applications, as can easily be seen from correlograms con-

57 sisting of a "causal" contribution, which contains the energy traveling from the station

${ }_{58}$ taken as the reference to the other, and an "anticausal" for the reverse case: causal and

${ }_{59}$ anticausal parts are symmetric with respect to the origin of the time axis only in presence of a diffuse wavefield. A diffuse wavefield can be generated if the noise sources are

${ }_{61}$ distributed uniformly or if scattering processes mimic the effect of this kind of distribu-

${ }_{62}$ tion. However, at the frequencies we consider in this study, scattering is not expected

${ }_{63}$ to be sufficient to randomize wave propagation directions [Paul et al., 2005]. Figure 1

${ }_{64}$ shows that often (depending on configuration and properties of sources, scattering, ab-

${ }_{65}$ sorption) the two branches of the correlograms present high asymmetry. Stehly et al.

${ }_{66}$ [2006], Kedar et al. [2008], Landès et al. [2010] and Hillers et al. [2012] show how the

${ }_{67}$ sources of ambient seismic noise, far from being uniform, are concentrated in the sea

${ }_{68}$ regions. In particular Stehly et al. [2006] verified, by analyzing the spectral bands corre-

${ }_{69}$ sponding to the primary (10-20 s) and the secondary (5-10 s) microseism for three different 
70 datasets of ambient noise correlograms (North America, Africa and Europe), how the sec-

71 ondary microseism is related to the interaction of the ocean swell with the coastlines,

72 while the primary microseism is related to ocean wave activity in deep water. Snieder

${ }_{73}$ [2004] suggests that source nonuniformity should not entirely compromise ambient-noise

${ }_{74}$ measurements of surface-wave velocity. However Tsai and Moschetti [2010] proved the

75 importance of source distribution effects on surface-wave amplitudes and Hanasoge [2012]

76 shows that the distribution of sources influences correlograms and how their knowledge

77 is important to correctly interpret these data. Mulargia [2012] after analyzing from a

78 statistical perspective the azimuthal isotropy of ambient-noise data recorded in various

79 parts of the world, concludes that seismic noise wavefield is not generally diffuse.

so The theoretical work of Tromp et al. [2010] (T10 hereafter) shows how adjoint techniques

${ }_{81}$ [e.g. Tromp et al., 2005; Peter et al., 2007] can be applied to ambient-noise seismology,

${ }_{82}$ taking into account the nonuniform distribution of noise sources. Our work represents

${ }_{83}$ one of the first applications of the adjoint methodology to a large, continental-scale set

${ }_{84}$ of high-quality ambient-noise correlograms in Europe [Verbeke et al., 2012]. We shall

${ }_{85}$ highlight, in particular, the importance of properly defining the geographic distribution

${ }_{86}$ of noise sources, with the ultimate goal of improving on existing models of crust and

${ }_{87}$ uppermost mantle.

${ }_{88}$ The paper is organized as follows: in Section 2 we describe the background model for our

s9 European study area based on new crustal and tomographic models and its discretization

90 for 3D forward modeling. Section 3 describes the ambient-noise dataset gathered from this

${ }_{91}$ region of interest. In Section 4 we describe the different steps of the algorithm followed

${ }_{92}$ in this work; an Appendix offers a theoretical basis for the technique, largely based on 
93 T10. Section 5 shows sensitivity kernels upon source location and wavespeed structure

94 dependent on different starting models of the spatial distribution of noise sources, and

${ }_{95}$ different geographic distributions of seismic stations. From the analysis of the influence

96 that these parameters have on the sensitivity kernels we can obtain a quantitative insight

${ }_{97}$ into the origin of ambient-noise and its effect on inversions for 3D structure.

\section{Background model}

98 Seismic waveform tomography aims at minimizing a misfit between synthetic and observed seismic waveform to improve the quality of the structural model used to compute synthetic data. Considering the crustal sensitivities of surface waves in the ambient-noise period range, we start with a tomographic 3D model to partially circumvent issues such as cycle skips or irretrievably disparate waveforms that may appear in simpler scenarios. The 3D velocity model used in this work combines a high-resolution European crustal model EPcrust [Molinari and Morelli, 2011] with an adaptive-resolution tomographic upper-mantle model FMADVOXEU' [Schaefer et al., 2011].

EPcrust is derived from the collection of several earlier, independent studies ([e.g. Tesauro et al., 2008; Grad and Tiira, 2009; Stehly et al., 2009]) based on active seis- 
115 voxel parametrization in which the size of each parametrization pixel depends on the data ${ }_{116}$ coverage at and around the pixel. This way, model resolution reflects data coverage, and ${ }_{117}$ the model's information content is optimized without over- and/or underparameterization.

${ }_{118}$ In particular, parametrization is denser over the European region, where data coverage is ${ }_{119}$ better. Starting from values of shear wavespeed $\beta$ defined in the model, we derive values ${ }_{120}$ of compressional wave speed $\alpha$ and density $\rho$ using the relation [Karato, 2003]:

$$
R_{\beta / \alpha} \equiv \frac{\delta \log \beta}{\delta \log \alpha}
$$

122 and

$$
R_{\rho / \beta, \alpha} \equiv \frac{\delta \log \rho}{\delta \log (\beta, \alpha)}
$$

${ }_{124}$ where the variations of $\alpha$ and $\beta$ are with respect to the PREM model and $R$ has a ${ }_{125}$ value of 0.5 . One of the most important factors affecting the accuracy of numerical wave ${ }_{126}$ propagation modelling is the construction of a high-quality mesh. The spectral-element ${ }_{127}$ package SPECFEM3D has the capability to incorporate fully unstructured hexahedral ${ }_{128}$ meshes [Peter et al., 2011] using external meshers such as CUBIT [Blacker et al., 1994], 129 thus allowing for explicitely honoring all geological features (undulating Moho, surface ${ }_{130}$ topography) represented in models such as EPcrust.

${ }_{131}$ We built a mesh that covers Europe and the surrounding oceans at latitudes between ${ }_{132} 30^{\circ}$ and $65^{\circ}$ north and longitudes between $-46^{\circ}$ and $47^{\circ}$, with horizontal dimensions of ${ }_{133} 3000 \times 3000 \mathrm{~km}$ and a depth of $165 \mathrm{~km}$. The mesh honors Earth's curvature and topography ${ }_{134}$ as well as lateral variations in Moho depth (figure 2 (b)) according to EPcrust. At the ${ }_{135}$ period range $8-30 \mathrm{~s}$ considered in this work, surface waves have wavelength between ${ }_{136} 16$ and $24 \mathrm{~km}$, therefore, even if the topography is reproduced by the mesh, its effects will not be substantial in our simulations. The mesh is designed to simulate surface- 
${ }_{138}$ wave propagation at periods as low as $8 \mathrm{~s}$, containing 192,892 elements for a total of

\section{Cross-correlation measurements}

We use a carefully processed dataset of vertical component ambient-noise correlograms from around Europe [Verbeke et al., 2012]. This database has been obtained from one year of continuous seismic recording between January and December 2006 from the Swiss Network, the German Regional Seismological Network, the Italian national broad-band network and Orfeus. Using one year of data, seasonal effects associated with the geography of ocean storms are minimized [Stehly et al., 2006]. Correlograms are computed following the approach described in Bensen et al. [2007], Boschi et al. [2012] and Stehly et al. [2006].

After rejecting station couples with relatively low signal-to-noise ratio, the database includes 864 correlogram observations. We measure the traveltime difference between observation-based and synthetic correlograms within time windows centered on surface waves, i.e. one per correlogram. Before performing this measurement we apply a bandpass Butterworth filter to both synthetics and data considering different period ranges: the upper bandpass limit is fixed at $30 \mathrm{~s}$ while the lower limit takes values of 10,15 or $20 \mathrm{~s}$. 


\section{Computational procedure}

Whenever the normalized cross-correlation between synthetic and data falls below 0.5 , we ignore this measurement. Although this threshold value is low it is reasonable if we consider that (i) we are at the very first stage of the inversion process, (ii) the quality of the models used varies strongly within the area of study, (iii) considering such a wide area leads to a very high dispersion for surface waves so that an increase in the misfit is plausible. The number of measurements we could obtain for a given station dramatically decreased when considering period ranges with lower limits below 20 s. For example, our database contains a total of 45 observation-based ambient-noise correlograms for station AIGLE. Computing the traveltime misfit within the period band $20-30 \mathrm{~s}$ we obtained a total of 24 measurements, but upon enlargening the period band down to $10 \mathrm{~s}$, the number of acceptable measurements reduced to 15. This result was expected since EPcrust is best suited to reproduce signals with period between 20 and $30 \mathrm{~s}$ [Molinari et al., 2012], and the tomographic model at even longer period.

In Figure 4, we show examples of comparison between observation-based and synthetic ambient-noise correlograms for two pairs of seismic stations. The comparison for station couple AIGLE-CEY might indicate that EPcrust is overall too slow with respect to real Earth structure in this area. Station couple ZUR-TNS on the contrary shows a very good agreement between synthetic and observation-based ambient-noise correlogram.

T10 explain how the adjoint method [e.g., Tromp et al., 2005; Peter et al., 2007] can be applied in ambient-noise seismology to determine the sensitivity of noise correlograms to Earth and source properties, in particular for non-uniform distributions of noise-sources. 
${ }_{204}$ noise correlograms. We store the result of this first simulation at all points within the

${ }_{205}$ source region defined previously (see fig. 5 first column). The result of the first simulation

${ }_{206}$ is now used as forcing term in the second one, from which we obtain the wavefield $\boldsymbol{\Phi}^{\alpha}(\mathbf{x}, \omega)$

207 of equation (A14 (second column of figure 5).

${ }_{208}$ The cross-correlation traveltime difference acts as misfit between synthetic and observation-based ambient-noise correlograms:

$$
\Delta T^{\alpha \beta}=T_{s i m}^{\alpha \beta}-T_{o b s}^{\alpha \beta}
$$

${ }_{211}$ where $\Delta T^{\alpha \beta}$ relates to the stations pair $\alpha \beta$ (green and yellow dot in figure 5) and is ${ }_{212}$ determined by a cross-correlation of the synthetic and observation-based correlograms, ${ }_{213}$ for the same stations pair $T_{s i m}^{\alpha \beta}$ and $T_{o b s}^{\alpha \beta}$ denote the traveltime. Since both synthetic ${ }_{214}$ and observation-based ambient noise correlograms are dominated by surface waves, we 215 measure the misfit only in the time-window corresponding to the arrival of these wave 216 groups. For each synthetic correlogram, we select automatically the time window over ${ }_{217}$ which the traveltime difference is measured: the center of the interval is defined as the ${ }_{218}$ ratio of the distance between the station couple and the approximate minimum value of ${ }_{219}$ speed for Rayleigh waves. We select empirically for all the stations a fixed time-window ${ }_{220}$ width of $160 \mathrm{~s}$, so that the whole signal associated with surface waves will be included in 221 the misfit measurement.

The misfit is computed for each combination of reference and generic station, and following the same procedure used to obtain equation (A13) we define the adjoint sources ${ }_{224}$ for this kind of misfit as

$$
\mathbf{F}^{\dagger \alpha \beta}(\mathbf{x}, t)=-\frac{\hat{\nu}^{\beta} \Delta T^{\alpha \beta}\left\langle\dot{C}_{\text {sim }}^{\alpha \beta}\right\rangle(t) \delta\left(\mathbf{x}-\mathbf{x}^{\beta}\right)}{\int\left[\left\langle\dot{C}_{\text {sim }}^{\alpha \beta}\right\rangle\right]^{2}},
$$


${ }_{226}$ where $\hat{\nu}^{\beta}$ is the unit vector denoting the component of the station used for the correlogram.

We select 0.5 as the minimum value of the correlation coefficient between synthetic and

228

$$
\left\langle K_{\rho}^{\alpha \beta}\right\rangle=-\int \rho \boldsymbol{\Phi}^{\dagger \alpha \beta}(-t) \cdot \partial_{t}^{2} \boldsymbol{\Phi}^{\alpha}(t) \mathrm{d} t .
$$

In the third and final simulation we inject the adjoint force defined in equation (4) at the position of station $\beta$ (figure 5 third column, first panel). At the same time, we reconstruct the wavefield of the second simulation and the interaction of these two wavefields (figure 5 third column, second panel) described by equation (5) produces the "causal" sensitivity kernel for the density (figure 5 third column, third panel). The completion of the three simulations described above takes $1.3 \mathrm{~h}$ on 324 processors for one reference station. In order to perform a complete tomographic inversion, one needs to run this scenario for each reference station and iteration.

It is possible to define other types of kernels: for example in an isotropic Earth, we define the "causal" part of isotropic kernels for shear and bulk moduli respectively as

$$
\begin{aligned}
\left\langle K_{\mu}^{\alpha \beta}\right\rangle & =-\int 2 \mu\left[\mathbf{D}^{\dagger \alpha \beta}(-t): \mathbf{D}^{\alpha}(t)\right] \mathrm{d} t, \\
\left\langle K_{\kappa}^{\alpha \beta}\right\rangle & =-\int \kappa\left[\nabla \cdot \boldsymbol{\Phi}^{\dagger \alpha \beta}(-t) \nabla \boldsymbol{\Phi}^{\alpha}(t)\right] \mathrm{d} t,
\end{aligned}
$$

where the traceless strain deviator and its adjoint are given by

$$
\begin{gathered}
\mathbf{D}^{\alpha}=\frac{1}{2}\left[\nabla \boldsymbol{\Phi}^{\alpha}+\left(\nabla \boldsymbol{\Phi}^{\alpha}\right)^{T}\right]-\frac{1}{3}\left(\nabla \cdot \boldsymbol{\Phi}^{\alpha}\right) \mathbf{I}, \\
\left.\mathbf{D}^{\dagger \alpha \beta}=\frac{1}{2}\left[\nabla \boldsymbol{\Phi}^{\dagger \alpha \beta}+\left(\nabla \boldsymbol{\Phi}^{\dagger \alpha \beta}\right)^{T}\right)\right]-\frac{1}{3}\left(\nabla \cdot \boldsymbol{\Phi}^{\dagger \alpha \beta}\right) \mathbf{I} .
\end{gathered}
$$


${ }_{250}$ Using the two definitions of equation (6) it is possible to express the sensitivity kernels in ${ }_{251}$ terms of parameters such as the shear wave speed:

${ }_{252}\left\langle K_{\beta}^{\alpha \beta}\right\rangle=2\left[\left\langle K_{\mu}^{\alpha \beta}\right\rangle-\frac{4}{3} \frac{\mu}{\kappa}\left\langle K_{\kappa}^{\alpha \beta}\right\rangle\right]$.

${ }_{253}$ Finally, we define the source kernel, that represents the sensitivity for the location of ${ }_{254}$ ambient noise sources, as the convolution between the adjoint wavefield $\boldsymbol{\Phi}^{\dagger \alpha \beta}$ and the 255 forcing term $F^{\alpha}$ used in the second simulation:

$$
\left\langle K_{\sigma}^{\alpha \beta}\right\rangle=\int \boldsymbol{\Phi}^{\dagger \alpha \beta}(-t) \cdot \mathbf{F}^{\alpha}(t) \mathrm{d} t
$$

${ }_{257}$ Equations (5) - (11) can be obtained after some algebra from equation (A16).

The geographic distribution of noise sources $\sigma(\mathbf{x})$ is of fundamental importance in this

The complete kernels, based on the ensemble correlogram $\left\langle C^{\alpha \beta}\right\rangle(t)=\left\langle C^{\beta \alpha}\right\rangle(-t)$, can

$$
\langle K\rangle=\sum_{\alpha=1}^{N} \sum_{\beta \neq \alpha}\left\langle K^{\alpha \beta}\right\rangle
$$

266 which corresponds to a cumulative cost function

$$
\chi=\frac{1}{2} \sum_{\alpha=1}^{N} \sum_{\beta \neq \alpha} \Delta T^{\alpha \beta}
$$

Once the misfit kernel is assembled, it is possible to compute the gradient of the misfit ${ }_{269}$ function $g_{k}=\int_{\Omega} K B_{k} \mathrm{~d}^{3} \mathbf{x}$, where $\Omega$ is the model volume, $K$ is the misfit kernel and $B_{k}$ is a 270 set of basis functions used to parametrize the model and on to which we project the misfit ${ }_{271}$ kernel. This gradient can then be used to update the initial structural model within the 
${ }_{272}$ framework of an iterative inversion. Before updating the model, a general smoothing of

\section{Noise sensivitity kernels}


315 extending into the Cordillera Central in Spain. This high-sensitivity area protruding westward from station ARBF is presumably caused by the nonuniformity in the distribution 317 of noise sources: if sources were distributed uniformly, the sensitivity kernel would be ${ }_{318}$ reminiscent of classic "banana-doughnuts" [T10, figure (5)]. Oscillations and changes of 319 kernel values are probably caused by neglecting thin sediments as explained in Section 2, ${ }_{320}$ in fact figure 6 (c) represents the same $K_{\beta}$ of panel (b) but at a depth of $30 \mathrm{~km}$ and it 321 can be easily observed how the kernel at this depth is much smoother than above.

${ }_{322}$ In figure $6(\mathrm{~d})$ we plot the source sensitivity kernel $K_{\sigma}$, for the same couple of stations: ${ }_{323}$ the area between the two seismic stations does not show any sensitivity. The two areas 324 of sensitivity centered onto the seismic stations are different: the one pointing westward 325 from ARBF is larger and shows higher values compared to the one centered in KBA. ${ }_{326}$ Also, the sensitivity region centered in ARBF does not have the same hyperbolic shape ${ }_{327}$ as the one centered on KBA, i.e. the low branch of the hyperbola that, based on the 328 theoretical analysis of T10, one might expect to appear in the Mediterranean is missing. ${ }_{329}$ Another important feature is that the left lobe of the kernel presents a strong asymmetry 330 towards the Biscay bay and the Atlantic ocean. These features are a direct consequence 331 of the more effective interaction that the adjoint wavefield has with the forward wavefield coming from the Atlantic Ocean: these stations mostly record ambient noise coming from

The geographic orientation of the AQU-SSY (figure 7 (a)) is almost orthogonal to ARBF-KBA, and roughly north-south. In this configuration, the two stations record synthetic ambient noise coming from the North Sea as well as from the Mediterranean: in this case, the assumption of uniform noise-sources distribution is presumably more 
338 valid than for stations ARBF-KBA, in which the energy came primarly from the Atlantic

339 Ocean. This is reflected also in the shape of the sensitivity kernels. At the free surface

340 of our model (figure $7(\mathrm{~b})$ ), the $K_{\beta}$ sensitivity associated with AQU-SSY is concentrated

${ }_{341}$ between the two stations, in a small area around them and there is a prominent lobe of ${ }_{342}$ sensitivity centered on station AQU and pointing towards the Alps. The remarks about 343 oscillations in $K_{\beta}$ we made for ARBF-KBA are still valid, as shown by figure 7 (c). In 344 this case $K_{\sigma}$ (figure $7(\mathrm{~d})$ ), shows a higher symmetry than in the previous configuration ${ }_{345}$ and it is possible to see the classic hyperbolic shape of the two "jets", departing from the ${ }_{346}$ two seismic stations [Tromp et al., 2010].

\subsection{Different Distribution of Noise Sources}

${ }_{347}$ In this Section we discuss sensitivity kernels obtained for a subset of selected 26 seismic stations, and for three different scenarios of ambient noise sources distributions. For each scenario we follow the method described in Appendix A: we consider each of the 35026 stations respectively as the reference station, compute the sensitivity kernels for each ${ }_{351}$ reference station, and sum them together similar to summing event kernels as in Tape et al. ${ }_{352}^{32}$ [2007]. Our database of observation-based ambient-noise correlograms contains a different 353 number of data for each station of reference, but sensitivity kernels are not comparable ${ }_{354}$ with those shown in the previous Section, since they result from the interaction of many 355 singular kernels.

\subsubsection{Baltic Sea}

We first assume that all noise is generated in the Baltic Sea, i.e. the region covered ${ }_{358}$ by concentric grey circles in figure 8 . Plots of $K_{\sigma}$ in figure 8 (a) (b) and (c) show that certain stations then have a larger influence on the final results with respect to others: for 
${ }_{360}$ example we can see how kernel values obtained with ARSA as station of reference (figure

${ }_{361} 8(\mathrm{a})$ ) are larger than those obtained for stations such as ARCI or BSSO (respectively

362 figures 8 (b) and (c)). We sum together all these single contributions in order to obtain

${ }_{363}$ the gradient of the misfit function. The result of this sum is shown in figure 8 (d), from

364 which we can see how the source sensitivity kernels are largest along long and narrow

365 strips pointing towards three distinct regions: the Mediterranean sea (particularly in the

${ }_{366}$ Adriatic), the Atlantic (particularly towards the coasts of Portugal and Bay of Biscay),

${ }_{367}$ and eastern Europe. This last result is partially in contrast with the assumption we made

${ }_{368}$ about the location of ambient noise sources, that is to say noise originates principally

${ }_{369}$ in the sea. From the analysis of the source sensitivity kernel $K_{\sigma}$ for station ARSA, we

370 can notice the presence of a strong jet of sensitivity pointing eastwards. Since station

${ }_{371}$ ARSA is located eastward with respect to the other stations used to build this kernel, it

372 is straightforward to associate this result with this particular geographical position. The

${ }_{373} K_{\sigma}$ kernel associated with station BRANT, figure 9 , shows a lobe of sensitivity pointing

${ }_{374}$ towards east, even if in this case the reference station is central with respect to the ones

375 used to construct the sensitivity kernel.

376

377

378

379 


\subsection{Shear wavespeed sensitivity}


${ }_{428}$ ometry. Figure 12 (c) shows noise in all surrounding seas, resulting in a smoother, multi-

${ }_{429}$ directional wavespeed kernel covering a larger continental area. This may of course simply

${ }_{430}$ be due to the same factor as above, i.e. reflecting the noise-source and station geometry.

${ }_{431}$ Assuming uniform coverage may therefore generally have the effect of smoothing gradients

${ }_{446}$ to the northeast part of Germany.

447 
${ }_{451}$ we used has values of shear wavespeed too high. The final panel of figure 13 shows a ${ }_{452}$ slice of the misfit kernel taken at a depth of $70 \mathrm{~km}$. We notice how the sensitivity is ${ }_{453}$ almost completely positive: this suggests that the velocity model at this depth needs to ${ }^{454}$ be corrected with higher values of $\beta$. We are not expecting such monotone behaviour ${ }_{455}$ during the whole inversion process: in the first iteration of their inversion Tape et al. ${ }_{456}$ [2010] encountered a similar situation where the whole velocity model appeared to be ${ }_{457}$ too fast but, after some iterations the kernels showed a more balance in sign. Panel (d) ${ }_{458}$ of figure 13 shows how at $70 \mathrm{~km}$ depth the sensitivity areas become smoother and are ${ }_{459}$ characterized by lower values with respect to those at $10 \mathrm{~km}$ depth (panel (c) of the same ${ }_{460}$ figure): this is consistent with the fact that ambient noise comprises surface waves that ${ }_{461}$ propagate within the uppermost $150 \mathrm{~km}$.

${ }_{462}$ These kernels are the basis for an iterative inversion by means of which it will be possible ${ }_{463}$ to obtain a new tomographic image of Europe with much greater details than currently ${ }_{464}$ available, thanks to the high frequencies in the noise data.

\section{Conclusions}

We presented ambient-noise source distributions as an integral part of a tomographic

${ }_{466}$ inversion for crustal structure, and show the strong influence which a priori choices have ${ }_{467}$ on wavespeed gradients, the building blocks for tomographic inversions. We use a dataset ${ }_{468}$ of ambient-noise correlograms in central Europe [Verbeke et al., 2012] and analyze the ${ }_{469}$ sensitivity of noise-generated surface waves to 3D structure and the geographic distribu470 tion of the sources of ambient signal based on Tromp et al. [2010]. This framework allows ${ }_{471}$ for nonuniform noise-source distributions, to account for the effects of nonuniformity in ${ }_{472}$ noise-source distribution, which has not been possible in most ambient-noise tomography 
${ }_{473}$ literature so far. This is relevant to the problem of identifying the origin of ambient noise, ${ }_{474}$ closely related to the areas where noise comes from: for instance Yang et al. [2008] relate ${ }_{475}$ ambient noise to solid-earth-ocean coupling near coast, while Stehly et al. [2006] Hillers ${ }_{476}$ et al. [2012] and Landès et al. [2010] find thata it could also be generated by open-ocean ${ }_{477}$ processes. The workflow presented in Section 4 is general and can be applied to both local ${ }_{478}$ or global scale.

As a starting model, we use crustal model EPcrust [Molinari and Morelli, 2011] and a 3D

${ }_{484}$ kernels based on this misfit.

${ }_{485}$ We analyzed the influence of stations distribution on sensitivity kernels, in particular we 
${ }_{496}$ the position of the two stations, while in the east-west configuration this symmetry is lost. 
${ }_{519}$ sources other than the ones located in the sea or to the presence of a scatterer not resolved ${ }_{520}$ at present by the structural model used in this work.

\section{Appendix A: Outline of the theory}

We start out with the equation of motion

$$
\rho \partial_{t}^{2} \mathbf{s}(\mathbf{x}, t)-\nabla \cdot[\mathbf{c}(\mathbf{x}): \nabla \mathbf{s}(\mathbf{x}, t)]=\mathbf{f}(\mathbf{x}, t)
$$

[e.g., Dahlen and Tromp, 1998], where $\rho$ denotes density, x location, $t$ time, s displacement, $\mathbf{c}$ the elastic tensor, and the forcing term $\mathbf{f}$ describes ambient-noise sources. We introduce here an operator $L$ such that eq. (A1) can also be written in the more compact form

$$
L \mathbf{s}(\mathbf{x}, t)=\mathbf{f}(\mathbf{x}, t)
$$


In the assumption (typical for ambient-noise seismology) of spatially uncorrelated noise ${ }_{541} \quad$ [e.g., Boschi et al., 2012], the components $f_{1,2,3}$ of $\mathbf{f}$ have the property

$$
\left\langle f_{i}\left(\mathbf{x}^{\prime}, \omega\right) f_{j}\left(\mathbf{x}^{\prime \prime}, \omega\right)\right\rangle=S_{i j}\left(\mathbf{x}^{\prime}, \omega\right) \delta\left(\mathbf{x}^{\prime}-\mathbf{x}^{\prime \prime}\right) \quad(i=1,2,3 ; j=1,2,3)
$$

543 
${ }_{562}$ between the theoretical and observed ensemble correlograms $\left\langle C_{\text {sim }}^{\alpha \beta}\right\rangle$ and $\left\langle C_{\text {obs }}^{\alpha \beta}\right\rangle$, respec-

$$
\chi=\frac{1}{2} \int\left[\left\langle C_{s i m}^{\alpha \beta}\right\rangle-\left\langle C_{o b s}^{\alpha \beta}\right\rangle\right]^{2} \mathrm{~d} t-\left\langle\iint \boldsymbol{\lambda} \cdot(L \mathbf{s}-\mathbf{f}) \mathrm{d}^{3} \mathbf{x} \mathrm{d} t\right\rangle,
$$

be minimum, consistent with eq. (19) of T10. Notice that, while the second term at the right-hand side of (A5) is defined by the physics of the medium of propagation, the first term represents one possible choice of the misfit function used to compare data and model predictions. The sensitivity functions obtained in the following depend on such choice, and if misfit is defined differently, sensitivity functions must be corrected accordingly.

The variables against which we optimize eq. (A5) are the components of the perturbation $\delta \mathbf{s}(\mathbf{x}, t)$ to the displacement field. In their appendix A, T10 find an analytical expression for the variation

$$
\begin{array}{r}
\delta\left\{\int\left[\Delta\left\langle C^{\alpha \beta}\right\rangle\right]^{2} \mathrm{~d} t\right\}=\left\langle\int \int \left[\hat{\nu}^{\beta} \int \Delta\left\langle C^{\alpha \beta}\right\rangle(\tau) s^{\alpha}(t+\tau) d \tau \delta\left(\mathbf{x}-\mathbf{x}^{\beta}\right)\right.\right. \\
\left.\left.+\hat{\nu}^{\alpha} \int \Delta\left\langle C^{\beta \alpha}\right\rangle(\tau) s^{\beta}(t+\tau) \delta\left(\mathbf{x}-\mathbf{x}^{\alpha}\right)\right] \cdot \delta \mathbf{s}(\mathbf{x}, t) d^{3} \mathbf{x} d t\right\rangle
\end{array}
$$

5 of the first integral at the right-hand side of (A5) caused by small perturbations $\delta \mathbf{s}(\mathbf{x}, t)$.

In their appendix B, they likewise find the variation of the second integral at the righthand side of (A5) also resulting from $\delta \mathbf{s}(\mathbf{x}, t)$. Both contributions to $\delta \chi$ are combined in an expression that we write compactly as

$$
\begin{aligned}
\delta \chi & =\delta\left\{\int\left[\Delta\left\langle C^{\alpha \beta}\right\rangle\right]^{2} \mathrm{~d} t\right\}+\iint \boldsymbol{\lambda} \cdot L \delta \mathbf{s}(\mathbf{x}, t) \mathrm{d} t \mathrm{~d}^{3} \mathbf{x} \\
& +\iint \boldsymbol{\lambda} \cdot \delta L \mathbf{s}(\mathbf{x}, t) \mathrm{d} t \mathrm{~d}^{3} \mathbf{x}-\iint \boldsymbol{\lambda} \cdot \delta \mathbf{f}(\mathbf{x}, t) \mathrm{d} t \mathrm{~d}^{3} \mathbf{x}
\end{aligned}
$$

(compare with eq. (B1) of T10). In the case of no medium and source perturbations, eq. (A7) is reduced to

$$
\delta \chi=\delta\left\{\int\left[\Delta\left\langle C^{\alpha \beta}\right\rangle\right]^{2} \mathrm{~d} t\right\}+\iint \boldsymbol{\lambda} \cdot L \delta \mathbf{s}(\mathbf{x}, t) \mathrm{d} t \mathrm{~d}^{3} \mathbf{x}
$$

$$
\text { January 25, 2013, 4:59pm }
$$


${ }_{586}$ After replacing $\delta\left\{\int\left[\Delta\left\langle C^{\alpha \beta}\right\rangle\right]^{2} \mathrm{~d} t\right\}$ with its expression (A6), and the algebra carried out

${ }_{588}(\delta \chi=0)$ if the multiplier $\boldsymbol{\lambda}$ satisfies

$$
\delta \chi=\delta\left\{\int\left[\Delta\left\langle C^{\alpha \beta}\right\rangle\right]^{2} \mathrm{~d} t\right\}-\iint \delta \mathbf{s} \cdot L \boldsymbol{\lambda} \mathrm{d} t \mathrm{~d}^{3} \mathbf{x}
$$

equivalent to eq. (B6) of T10, with the right-hand side $\mathbf{f}^{\dagger}$ given by eq. (B7) of T10. Importantly $\mathbf{f}^{\dagger}$ contains the variation $\delta\left\{\int\left[\Delta\left\langle C^{\alpha \beta}\right\rangle\right]^{2} \mathrm{~d} t\right\}$, and hence the observation $\left\langle C_{o b s}^{\alpha \beta}\right\rangle$. Comparing (A10) with (A2), $\mathbf{f}^{\dagger}$ is naturally interpreted as a forcing term (a source). Let us dub it "adjoint source". The solution $\boldsymbol{\lambda}$ to (A10) is the corresponding displacement field, or the "adjoint wavefield", which is usually denoted $\mathbf{s}^{\dagger}$.

Let us now perturb the parameters describing the medium of propagation (density, elastic tensor), and the source term $\mathbf{f}$. We substitute $\boldsymbol{\lambda}$ with $\mathbf{s}^{\dagger}$ in eq. (A7); since $\mathbf{s}^{\dagger}$ is a solution to (A10), we find

$$
\delta \chi=-\frac{1}{2 \pi}\left\langle\iint\left(-\omega^{2} \delta \rho \mathbf{s}^{\dagger} \cdot \mathbf{s}+\nabla \mathbf{s}^{\dagger}: \delta \mathbf{c}: \nabla \mathbf{s}-\mathbf{s}^{\dagger} \cdot \delta \mathbf{f}\right) \mathrm{d}^{3} \mathbf{x} \mathrm{d} \omega\right\rangle
$$

equivalent to eq. (C1) of T10.

In their Appendix C, T10 manipulate eq. (A11) to show how ambient-noise sensitiviy kernels for any given station pair can be calculated with three spectral-element simulations only. They start by substituting s in (A11) with the convolution of the Green's function $\mathbf{G}$ and the forcing term $\mathbf{f}$, and likewise $\mathbf{s}^{\dagger}$ with the convolution of $\mathbf{G}$ and $\mathbf{s}^{\dagger}$. The resulting expression (eq. (C4) in T10) involves the products $f_{i}^{*}(\mathbf{x}, \omega) f_{j}\left(\mathbf{x}^{\prime}, \omega\right), f_{i}^{*}(\mathbf{x}, \omega) \delta f_{j}\left(\mathbf{x}^{\prime}, \omega\right)$ 


$$
\begin{gathered}
F_{i}^{\alpha}(\mathbf{x}, \omega)=G_{j k}^{*}\left(\mathbf{x}, \mathbf{x}^{\alpha} ; \omega\right) \nu_{k}^{\alpha} S_{i j}(\mathbf{x}, \omega), \\
\mathbf{F}^{\dagger \alpha \beta}(\mathbf{x}, \omega)=\hat{\nu}^{\beta} \Delta\langle C\rangle^{\alpha \beta}(\omega) \delta\left(\mathbf{x}-\mathbf{x}^{\beta}\right),
\end{gathered}
$$

with associated wavefields (eqs. (C8) and (C11) of T10)

$$
\begin{gathered}
\boldsymbol{\Phi}^{\alpha}(\mathbf{x}, \omega)=\int_{V} \mathbf{G}\left(\mathbf{x}, \mathbf{x}^{\prime} ; \omega\right) \cdot \mathbf{F}^{\alpha}\left(\mathbf{x}^{\prime}, \omega\right) \mathrm{d}^{3} \mathbf{x}^{\prime}, \\
\boldsymbol{\Phi}^{\dagger \alpha \beta}(\mathbf{x}, \omega)=\int_{V} \mathbf{G}\left(\mathbf{x}, \mathbf{x}^{\prime} ; \omega\right) \cdot \mathbf{F}^{\dagger \alpha \beta}\left(\mathbf{x}^{\prime}, \omega\right) \mathrm{d}^{3} \mathbf{x}^{\prime} .
\end{gathered}
$$

Substituting $\mathbf{F}^{\dagger \alpha \beta}, \mathbf{F}^{\alpha}, \boldsymbol{\Phi}^{\alpha}$ and $\boldsymbol{\Phi}^{\dagger \alpha \beta}$ into their eq. (C5) for the variation $\delta \chi$, T10 find the following compact expression

$$
\begin{aligned}
\delta \chi & =-\frac{1}{2 \pi} \iint\left[-\omega^{2} \delta \rho\left(\Phi_{i}^{\dagger \alpha \beta} \Phi_{i}^{\alpha}+\Phi_{i}^{\dagger \beta \alpha} \Phi_{i}^{\beta}\right)+\delta c_{i j k l}\left(\nabla_{i} \Phi_{j}^{\dagger \alpha \beta} \nabla_{k} \Phi_{l}^{\alpha}+\nabla_{i} \Phi_{j}^{\dagger \beta \alpha} \nabla_{k} \Phi_{l}^{\beta}\right)\right] \mathrm{d}^{3} \mathbf{x} \mathrm{d} \omega \\
& +\frac{1}{2 \pi} \iint\left(\Phi_{i}^{\dagger \alpha \beta} \delta F_{i}^{\alpha}+\Phi_{i}^{\dagger \beta \alpha} \delta F_{i}^{\beta}\right) \mathrm{d}^{3} \mathbf{x} \mathrm{d} \omega,
\end{aligned}
$$

where $\delta F_{i}^{\alpha}(\mathbf{x}, \omega)=G_{j k}^{*}\left(\mathbf{x}, \mathbf{x}^{\alpha} ; \omega\right) \nu_{k}^{\alpha} \delta S_{i j}(\mathbf{x}, \omega)$. It should be clear that the factors multiplying $\delta \rho, \delta c_{i j k l}$ and the combination of adjoint wavefield $\boldsymbol{\Phi}^{\dagger}$ with the variation of the force $\delta \mathbf{F}$ in (A16) are nothing but the partial derivatives (sensitivity kernels) relating our cost function to variations in the model parameters (density and elastic tensor) and source properties, which will be the unknowns of our inverse problem.

Now, in the same spirit as, e.g., Peter et al. [2007], the convolution of a Green's function with any source term can be interpreted as the result of injecting said source term in a numerical integration algorithm, like, in our case, the spectral element software of Tromp 
${ }_{627}$ et al. [2008], and computing the associated wavefield. Inspection of eq. (A16) shows that

\section{Appendix B: Validation of the spherical mesh}


${ }_{649}$ to eliminate all the possible numerical noise. This period range is the same in which the

synthetic noise used in our application is defined. The comparison of results obtained with the two different codes shows a good agreement both in terms signal phases and amplitudes.

\section{Acknowledgments.}

This work greatly benefitted from discussions with J. Tromp, D. Peter, and S. Hanasoge and from the comments of the two reviewers C. Tape and G. Hillers. This work has been supported by the European Marie-Curie initial training network QUEST and the Swiss HP2C platform project "Petaquake". Simulations were undertaken at the Swiss National Supercomputing Center, whose support is greatly appreciated.

\section{References}

Aki, K. (1957), Space and time spectra of stationary stochastic waves, with special reference to microtremors, B. Earthq. Res. I Tokyo, 35(3), 415-456.

Bensen, G. D., M. H. Ritzwoller, M. P. Barmin, A. L. Levshin, F. Lin, M. P. Moschetti, N. M. Shapiro, and Y. Yang (2007), Processing seismic ambient noise data to obtain reliable broad-band surface wave dispersion measurements, Geophys. J. Int., 169(3), 1239-1260, doi:10.1111/j.1365-246X.2007.03374.x.

Bijwaard, H., and W. Spakman (2000), Non-linear global P-wave tomography by iterated linearized inversion, Geophys. J. Int., 141(1), 71-82, doi:10.1046/j.1365246X.2000.00053.x.

Blacker, T. D., W. J. Bohnho, and T. L. Edwards (1994), CUBIT mesh generation environment. Volume 1: Users manual, Sandia National Labs., Albuquerque, NM (United 

States), doi:10.2172/10176386.

Boschi, L., B. Fry, G. Ekström, and D. Giardini (2009), The European Upper Mantle as Seen by Surface Waves, Surv. Geophys., 30(4-5), 463-501, doi:10.1007/s10712-0099066-2.

Boschi, L., C. Faccenna, and T. W. Becker (2010), Mantle structure and dynamic topography in the Mediterranean Basin, Geophys. Res. Lett., 37(20), 1-6, doi: 10.1029/2010GL045001.

Boschi, L., C. Weemstra, J. Verbeke, G. Ekström, and D. Giardini (2012), On measuring surface-wave phase velocity from station-station cross-correlation of ambient signal., submitted to Geophys. J. Int.

Campillo, M., and A. Paul (2003), Long-range correlations in the diffuse seismic coda, Science, 299(5606), 547-549, doi:10.1126/science.1078551.

Chang, S.-J., et al. (2010), Joint inversion for three-dimensional S velocity mantle structure along the Tethyan margin, J. Geophys. Res., 115(B8), 1-22, doi: 10.1029/2009JB007204.

Cox, H. (1973), Spatial correlation in arbitrary noise fields with application to ambient sea noise, J. Acoust. Soc. Am., 54(5), 1289-1301.

Dahlen, F., and J. Tromp (1998), Theoretical Global Seismology, Princeton University Press.

Duvall, T., S. Jeffferies, J. Harvey, and M. Pomerantz (1993), Timedistance helioseismology, Nature, 362(6419), 430-432.

Grad, M., and T. Tiira (2009), The Moho depth map of the European Plate, Geophys. J. Int., $176(1), 279-292$, doi:10.1111/j.1365-246X.2008.03919.x. 
Hanasoge, S. (2012), The influence of noise sources on cross-correlation amplitudes, Geophys. J. Int., submitted.

Hasselmann, K. (1963), A statistical analysis of the generation of microseisms, Rev. Geophys., 1(2), 177-210, doi:10.1029/RG001i002p00177.

Hillers, G., N. Graham, M. Campillo, S. Kedar, M. Landès, and N. Shapiro (2012), Global oceanic microseism sources as seen by seismic arrays and predicted by wave action models, Geochem. Geophy. Geosy., 13(1), Q01021, doi:10.1029/2011GC003875. Karato, S. (2003), The dynamic structure of the deep Earth, Princeton University Press. Kedar, S., M. Longuet-Higgins, F. Webb, N. Graham, R. Clayton, and C. Jones (2008), The origin of deep ocean microseisms in the North Atlantic Ocean, Proc. R. Soc. A, 464, 777-793, doi:10.1098/rspa.2007.0277.

Landès, M., F. Hubans, N. M. Shapiro, A. Paul, and M. Campillo (2010), Origin of deep ocean microseisms by using teleseismic body waves, J. Geophys. Res., 115, B05302, doi:10.1029/2009JB006918.

Longuet-Higgins, M. S. (1950), A theory of the origin of microseisms, Phil. Trans. R. Soc. A, 243, 1-35.

Mikesell, T. D., K. van Wijk, T. E. Blum, R. Snieder, and H. Sato (2012), Analyzing the coda from correlating scattered surface waves., J. Acoust. Soc. Am., 131(3), EL275-81, doi:10.1121/1.3687427.

Molinari, I., and A. Morelli (2011), EPcrust: a reference crustal model for the European Plate, Geophys. J. Int., 185(1), 352-364, doi:10.1111/j.1365-246X.2011.04940.x.

Molinari, I., J. Verbeke, L. Boschi, E. Kissling, and A. Morelli (2012), Mapping the Italian and Central Europe crust from a combination of ambient-noise and seismic 
data, Geochem. Geophy. Geosy., submitted.

Mulargia, F. (2012), The seismic noise wavefield is not diffuse., J. Acoust. Soc. Am., $131(4), 2853-8$, doi:10.1121/1.3689551.

Paul, A., M. Campillo, L. Margerin, E. Larose, and A. Derode (2005), Empirical synthesis of time-asymmetrical Green function from the correlation of coda waves, J. Geophys. Res., 110, B08302, doi:10.1029/2004JB003521.

Peter, D., C. Tape, L. Boschi, and J. H. Woodhouse (2007), Surface wave tomography: global membrane waves and adjoint methods, Geophys. J. Int., 171(3), 1098-1117, doi:10.1111/j.1365-246X.2007.03554.x.

Peter, D., et al. (2011), Forward and adjoint simulations of seismic wave propagation on fully unstructured hexahedral meshes, Geophys. J. Int., 186(2), 721-739, doi: 10.1111/j.1365-246X.2011.05044.x.

Peterson, J. (1993), Observations and modelling of background seismic noise.

Sabra, K. G., P. Gerstoft, P. Roux, W. A. Kuperman, and M. C. Fehler (2005), Surface wave tomography from microseisms in Southern California, Geophys. Res. Lett., 32, L14311, doi:10.1029/2005GL023155.

Schaefer, J. F., L. Boschi, T. W. Becker, and E. Kissling (2011), Radial anisotropy in the European mantle: Tomographic studies explored in terms of mantle flow, Geophys. Res. Lett., 38(23), 1-5, doi:10.1029/2011GL049687.

Schivardi, R., and A. Morelli (2009), Surface wave tomography in the European and Mediterranean region, Geophys. J. Int., 177(3), 1050-1066, doi:10.1111/j.1365246X.2009.04100.x. 
${ }_{738}$ Schmid, C., S. van der Lee, and D. Giardini (2004), Delay times and shear wave splitting in the Mediterranean region, Geophys. J. Int., 159(1), 275-290.

Shapiro, N. M., and M. Campillo (2004), Emergence of broadband Rayleigh waves from correlations of the seismic ambient noise, Geophys. Res. Lett., 31, L07614, doi: 10.1029/2004GL019491.

Shapiro, N. M., M. Campillo, L. Stehly, and M. H. Ritzwoller (2005), High-resolution surface-wave tomography from ambient seismic noise., Science, 307(5715), 1615-8, doi: 10.1126/science.1108339.

Snieder, R. (2004), Extracting the Greens function from the correlation of coda waves: A derivation based on stationary phase, Phys. Rev. E, 69(4), doi: 10.1103/PhysRevE.69.046610.

Stehly, L., M. Campillo, and N. M. Shapiro (2006), A study of the seismic noise from its long-range correlation properties, J. Geophys. Res., 111(B10), 1-12, doi: 10.1029/2005JB004237.

Stehly, L., B. Fry, M. Campillo, N. M. Shapiro, J. Guilbert, L. Boschi, and D. Giardini (2009), Tomography of the Alpine region from observations of seismic ambient noise, Geophys. J. Int., 178(1), 338-350, doi:10.1111/j.1365-246X.2009.04132.x.

Tanimoto, T. (2008), Normal-mode solution for the seismic noise cross-correlation method, Geophys. J. Int., 175(3), 1169-1175, doi:10.1111/j.1365-246X.2008.03959.x.

Tape, C., Q. Liu, and J. Tromp (2007), Finite-frequency tomography using adjoint methods-Methodology and examples using membrane surface waves, Geophys. J. Int., 168(3), 1105-1129, doi:10.1111/j.1365-246X.2006.03191.x. 
Tape, C., Q. Liu, A. Maggi, and J. Tromp (2010), Seismic tomography of the southern California crust based on spectral-element and adjoint methods, Geophys. J. Int., 180(1), 433-462, doi:10.1111/j.1365-246X.2009.04429.x.

Tesauro, M., M. K. Kaban, and S. A. P. L. Cloetingh (2008), EuCRUST-07: A new reference model for the European crust, Geophys. Res. Lett., 35(5), 1-5, doi: 10.1029/2007GL032244.

Tromp, J., C. Tape, and Q. Liu (2005), Seismic tomography, adjoint methods, time reversal and banana-doughnut kernels, Geophys. J. Int., 160(1), 195-216, doi:10.1111/j.1365246X.2004.02453.x.

Tromp, J., D. Komattisch, and Q. Liu (2008), Spectral-element and adjoint methods in seismology, Commun. Comput. Phys., 3(1), 1-32.

Tromp, J., Y. Luo, S. Hanasoge, and D. Peter (2010), Noise cross-correlation sensitivity kernels, Geophys. J. Int., 183(2), 791-819, doi:10.1111/j.1365-246X.2010.04721.x.

Tsai, V. C., and M. P. Moschetti (2010), An explicit relationship between time-domain noise correlation and spatial autocorrelation (SPAC) results, Geophys. J. Int., 182(1), 454-460, doi:10.1111/j.1365-246X.2010.04633.x.

Verbeke, J., L. Boschi, L. Stehly, E. Kissling, and a. Michelini (2012), High-resolution Rayleigh-wave velocity maps of central Europe from a dense ambient-noise data set, Geophys. J. Int., 188(3), 1173-1187, doi:10.1111/j.1365-246X.2011.05308.x.

Yang, Y., M. H. Ritzwoller, F.-C. Lin, M. P. Moschetti, and N. M. Shapiro (2008), Structure of the crust and uppermost mantle beneath the western United States revealed by ambient noise and earthquake tomography, J. Geophys. Res., 113(B12), 1-9, doi: 10.1029/2008JB005833. 
Zhou, Y., Q. Liu, and J. Tromp (2011), Surface wave sensitivity: mode summation versus adjoint SEM, Geophys. J. Int., 187(3), 1560-1576, doi:10.1111/j.1365246X.2011.05212.x.

Zhu, H., E. Bozda, D. Peter, and J. Tromp (2012), Structure of the European upper mantle revealed by adjoint tomography, Nature Geosci., 5(7), 493-498, doi:10.1038/ngeo1501. 
a)



c)

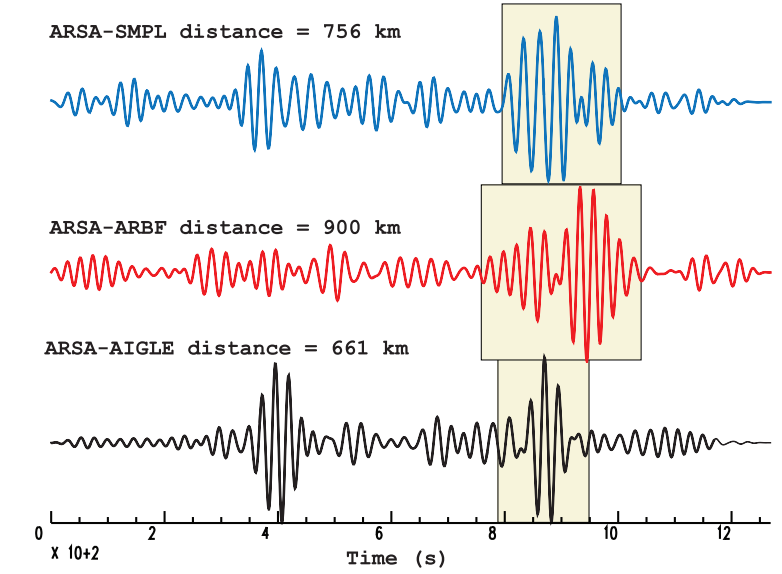

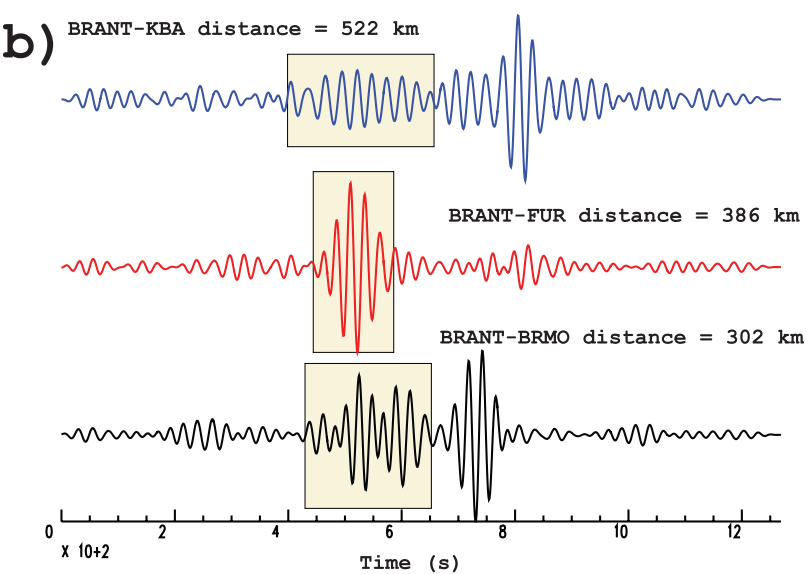

Figure 1. Set of correlograms (vertical components) from ambient noise recorded data, showing a certain portion of energy coming from east, all correlograms have been normalized to the unit so the vertical axis is not shown. (a) Positions of two sets (red dots and purple dots) of seismic stations. (b) Each time series represents a observation-based ambient noise correlogram with BRANT as reference station, the other stations used are respectively KBA (blue line), FUR (red line) and BRMO (black line). Since the reference station is located westward with respect to the others (panel (a)), surface waves traveling from east are visible in the left part, the anticausal one, of these correlograms. (c) In this case the reference station is ARSA and the one used to compute the correlograms are SMPL (blue line), ARBF (red line) and AIGLE (black line). The reference station is positioned eastward with respect to the others (panel (a)), thus it is possible to recognize the energy of surface waves propagating from east in the shaded boxes in the right part of the correlograms. Data in panel (b) and (c) are filtered using a bandpass Butterworth filter Abqtween 20 and $30 \mathrm{~s}$. 
a)

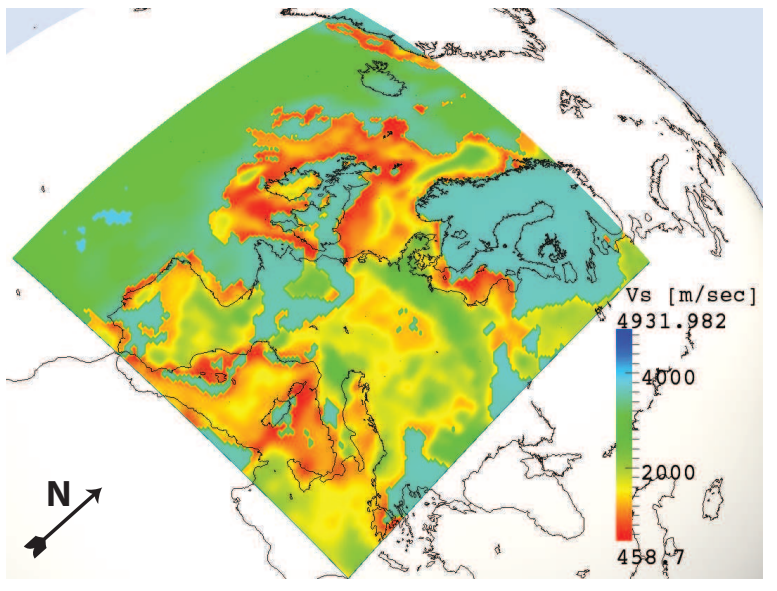

c)



b)



d)

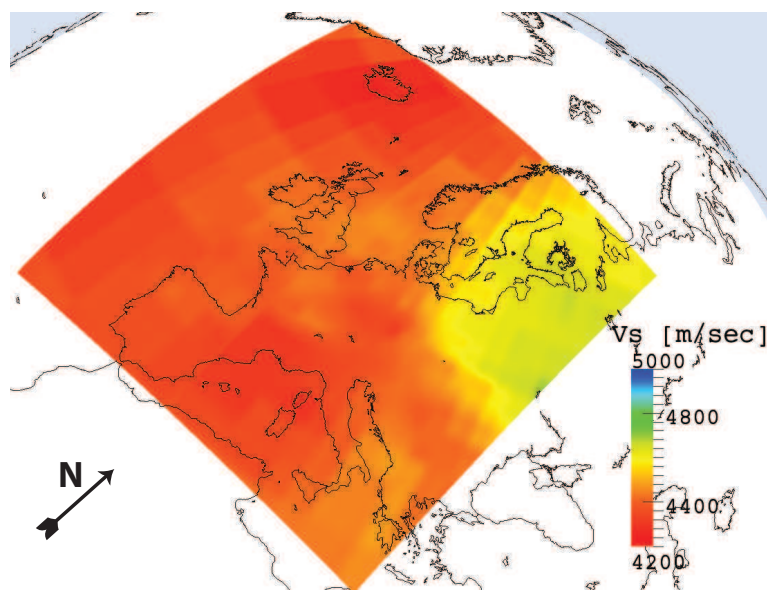

Figure 2. (a) Map for shear waves speed values of model EPcrust just below the free surface. Low velocity values are typical of sedimentary basins. Observed contrasts in speed values are caused by the absence of sediments in certain areas of our study region. (b) 3D view of the Moho surface geometry using an exaggeration factor of 10 to enhance the perspective. (c) Map for shear waves speed values of model FMADVOXEU' at a depth of $78 \mathrm{~km}$. (d) Map for shear waves speed values of model FMADVOXEU' at a depth of $128 \mathrm{~km}$. 




Figure 3. Close view of a vertical cross-section of the mesh used in this work. Topography is present but not visible at this one-to-one scale. The crustal layer is reproduced by means of two spectral elements. Notice how the irregular shape of the Moho produces a strong squeezing of the elements. This factor drives the stability condition of our simulations. 
a)
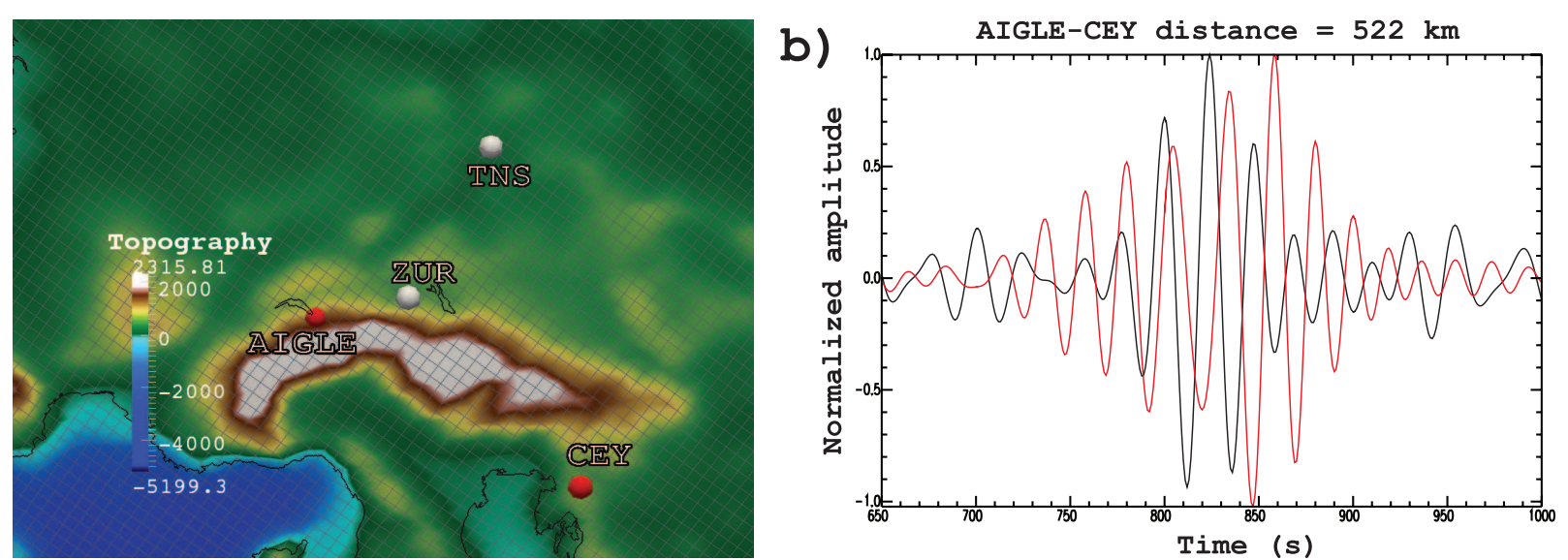

c)

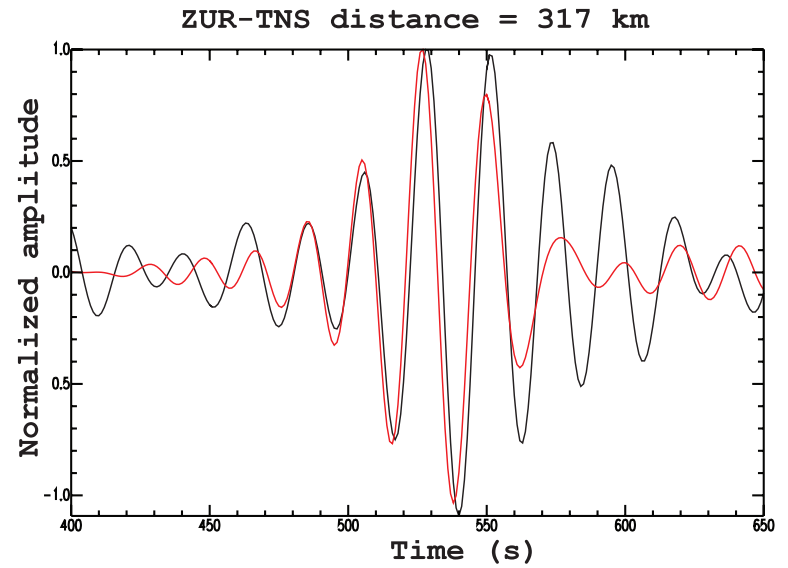

Figure 4. (a) Position of the two station couples. (b) and (c) comparison between correlograms from observation-based (black line) and synthetic (red line) traces. The unacceptable misfit between data and synthetic in panel (b) indicates how the model we used does not describe the complexity of the Alpine region very well. Synthetic correlograms are obtained following the methodology described in Section 4 and outlined in figure 5. 

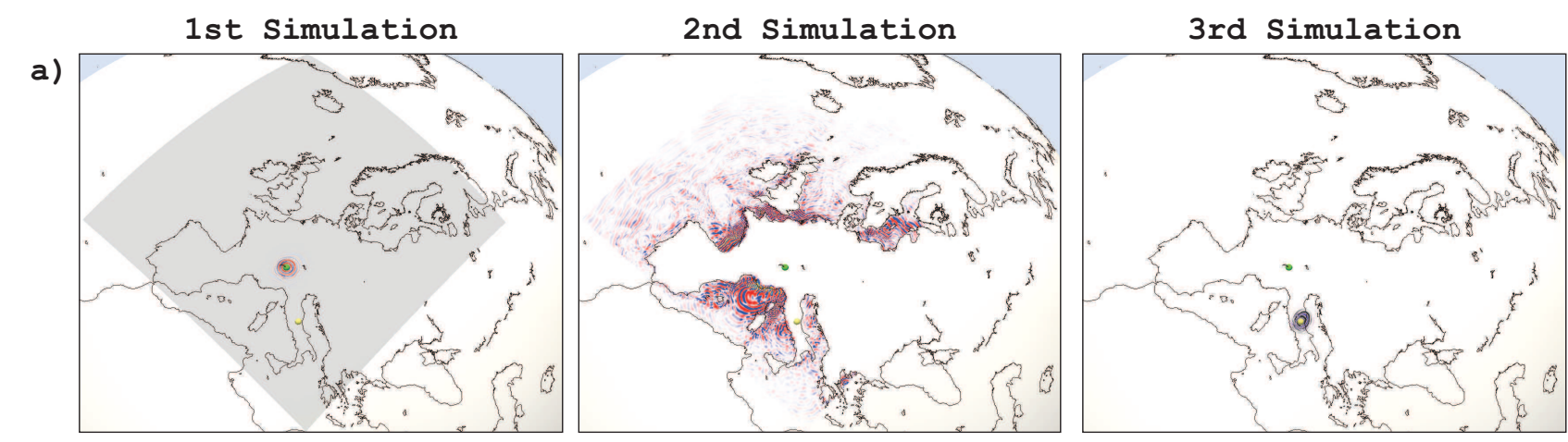

b)
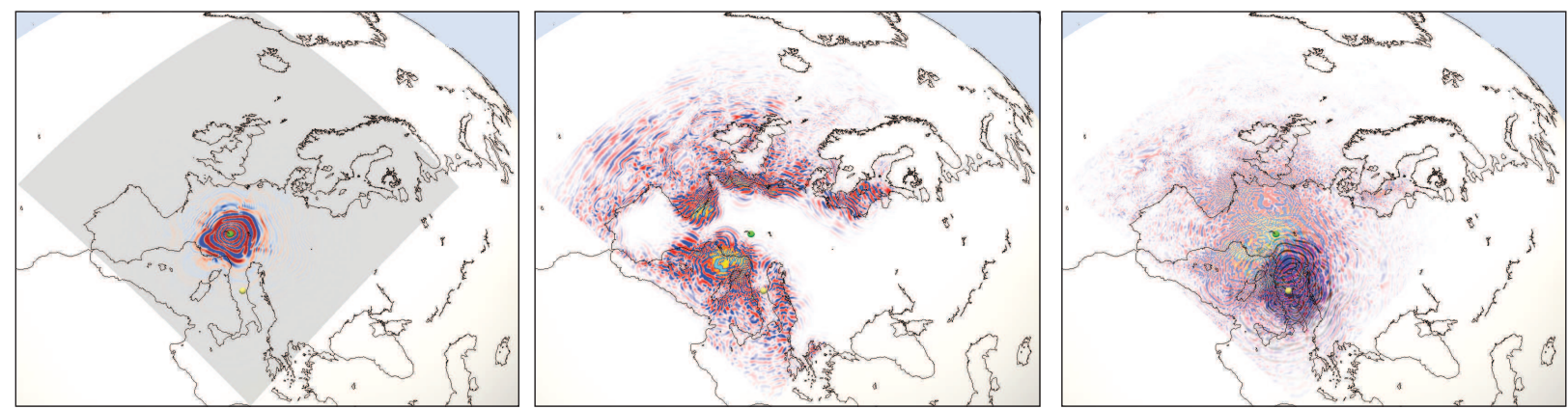

c)
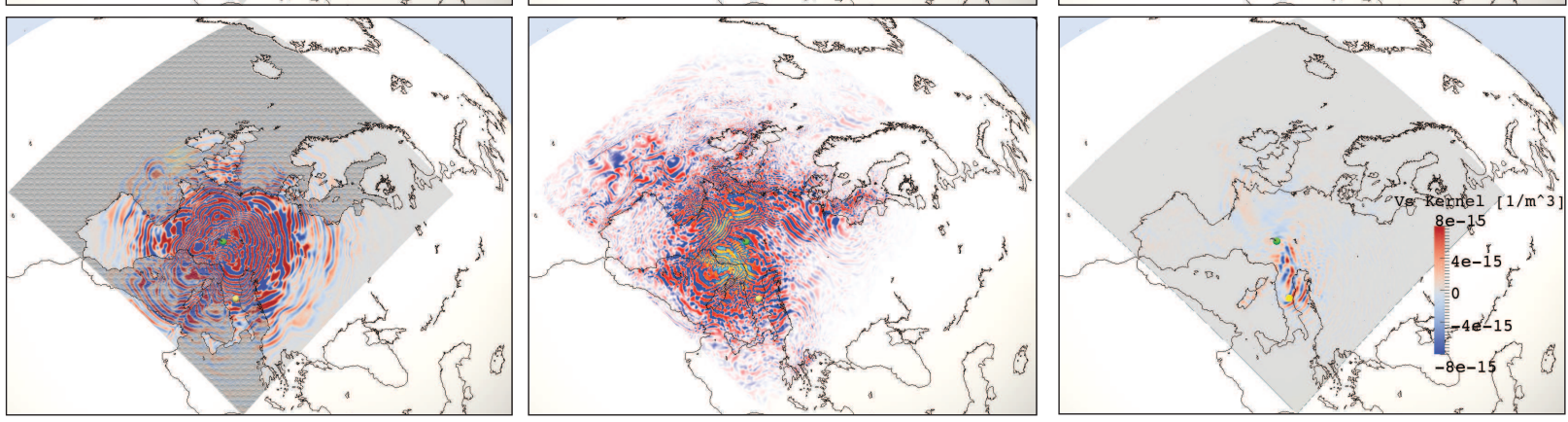

Figure 5. Synoptic scheme of the three simulations described in Section 4. Each column reproduces the subsequent steps of a simulation. The green dot represents the reference station AIGLE (station $\alpha$ in Section 4), while station AQU (station $\beta$ in Section 4) is indicated by the yellow dot. First column: three snapshots of the wavefield of the first simulation, produced by the forcing term $F_{i}^{\alpha}$ defined in equation (A12). Dark grey dotted area in the bottom left panel indicates the region where we guess all the noise sources are located and where the wavefield of the first simulation is stored. Second column: three snapshot of the second simulation where the wavefield stored from column (1) is used as forcing term and the result is the wavefield $\mathbf{\Phi}^{\alpha}(\mathbf{x}, \omega)$ of equation (A14). Third column (a)-(b): adjoint wavefield driven by eq.(4) and its interaction with the reconstructed field of the second simulation. Bottom right panel: picture of the "causal" $\beta$ sensitivity kernel eq. (5). 
a)

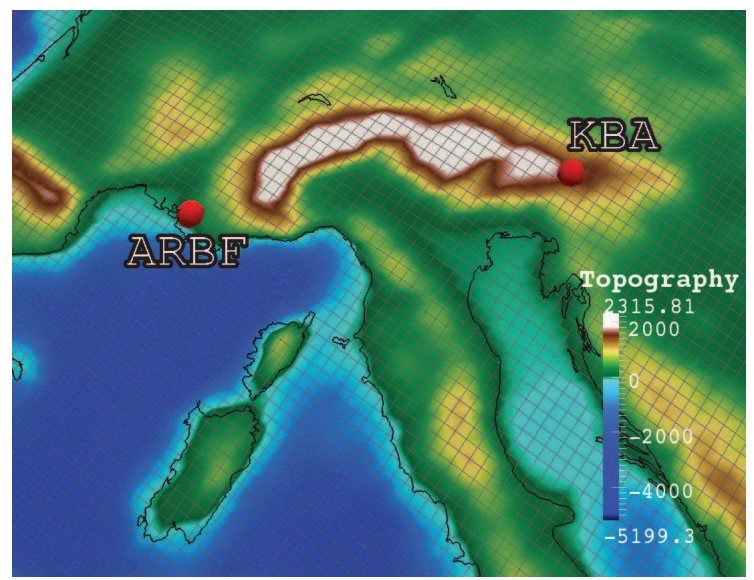

C)

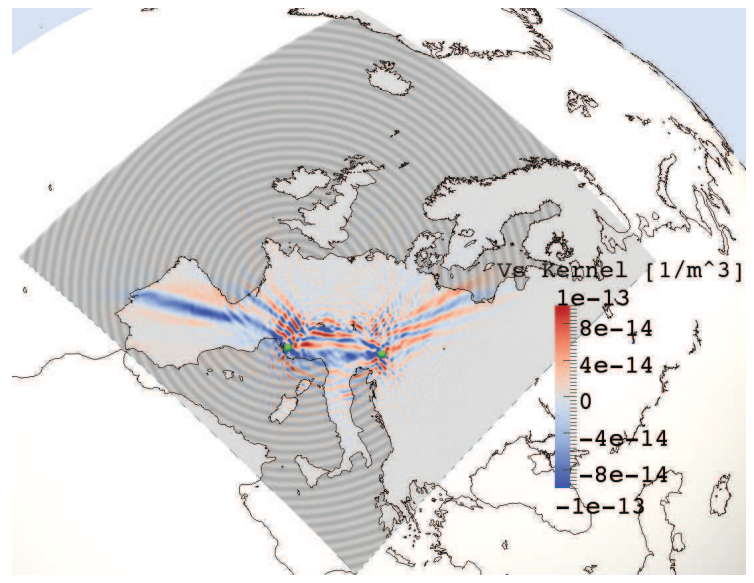

b)

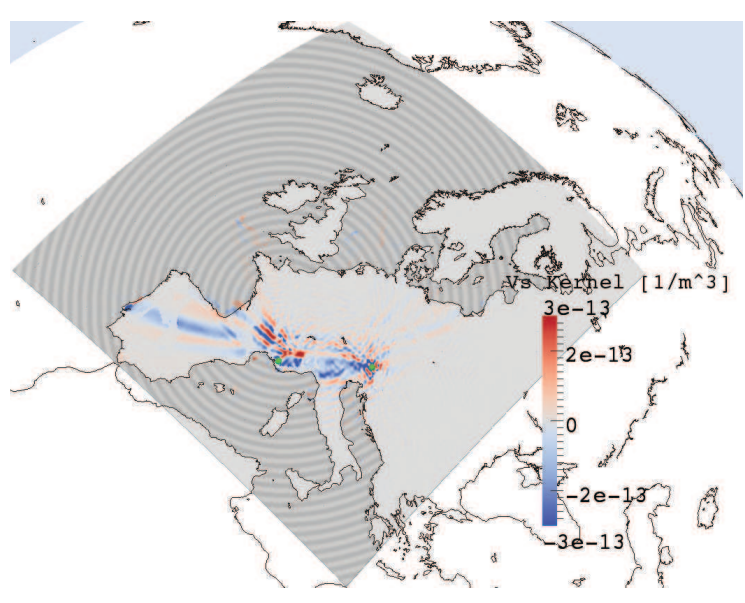

d)



Figure 6. Demonstrating the effect of uniform ocean noise sources, represented by the grey circles, on the $\beta$ and source sensitivity kernels. (a) Locations of stations ARBF (left red dot) and KBA (right red dot). (b) Shear wavespeed kernel for station pair ARBF-KBA just below the free surface. Sensitivity extends also outside the region between the two stations: this behavior is caused by the non uniform distribution of noise sources. (c) Same kernel showed in panel (b), but at a depth of $30 \mathrm{~km}$. Owing to the absence of sediments at this depth, the kernel is smoother. (d) Source kernel. The two areas of sensitivity centered onto the seismic stations have interesting features: (i) the one pointing westward shows higher values than the one pointing eastward, and (ii) it has not the same hyperbolic shape of the one centered on KBA (sensitivity in the Mediterranean is near zero). These features indicate that most of the energy originates in the Atlantic. 
a)

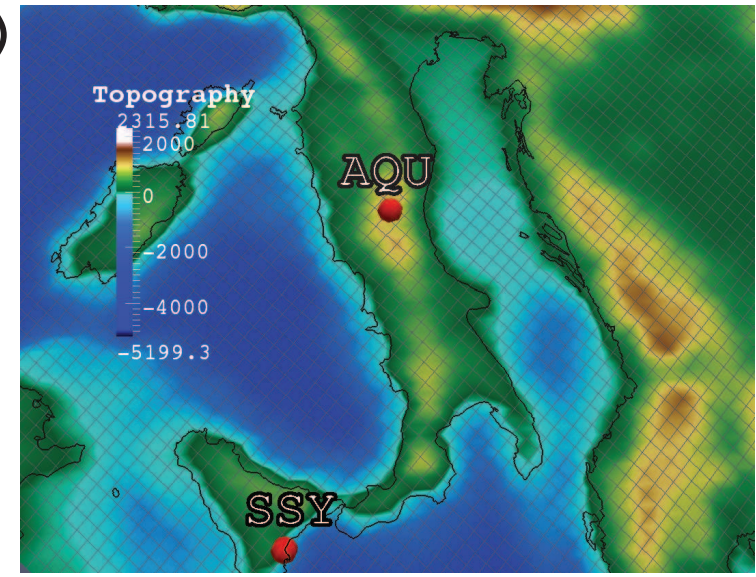

c)



b)

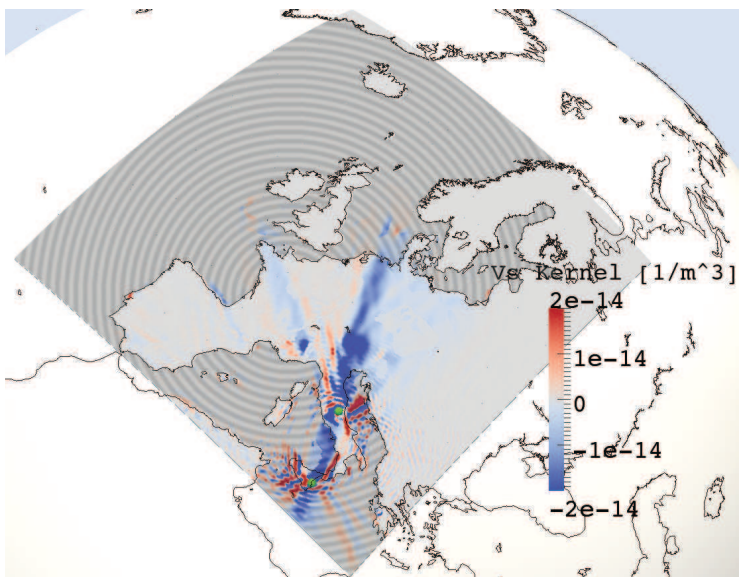

d)

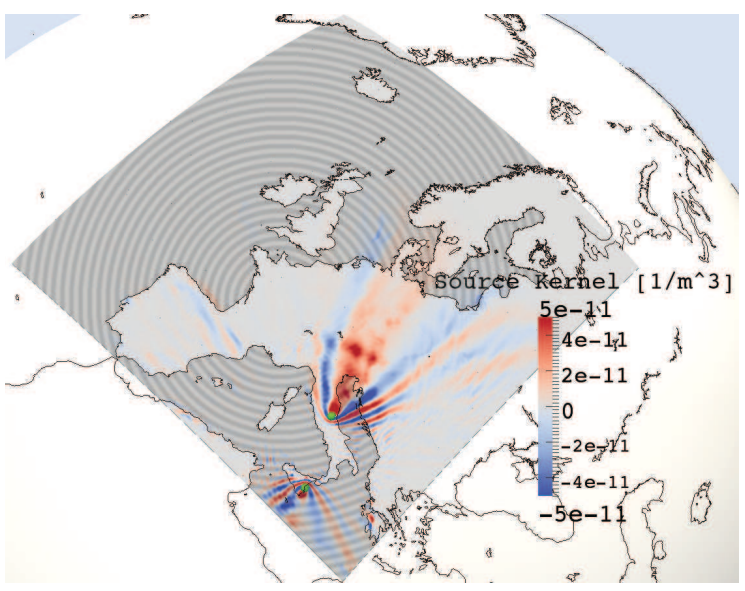

Figure 7. (a) Locations of stations AQU (center Italy) and SSY (Sicily). Synthetic ambient noise sources are placed in all seas and are represented by the grey circles. (b) $K_{\beta}$ for station-pair AQU-SSY just below the free surface. The sensitivity is concentrated between the two stations and in a small area around them with three "jets" departing from station AQU: the first one points towards the Alpine region while the other two are directed towards Croatia. (c) Slice of $K_{\beta}$ taken at a depth of $30 \mathrm{Km}$. Considerations made for panel (c) in figure 6 are also valid in this case. (d) The high symmetry shown by $K_{\sigma}$ is caused by the north-south orientation of the stations pair: synthetic ambient noise comes from the north (North Sea) as well from the south (Mediterranean), thus this situation is similar to a uniform distribution of noise sources. 
a)



c)

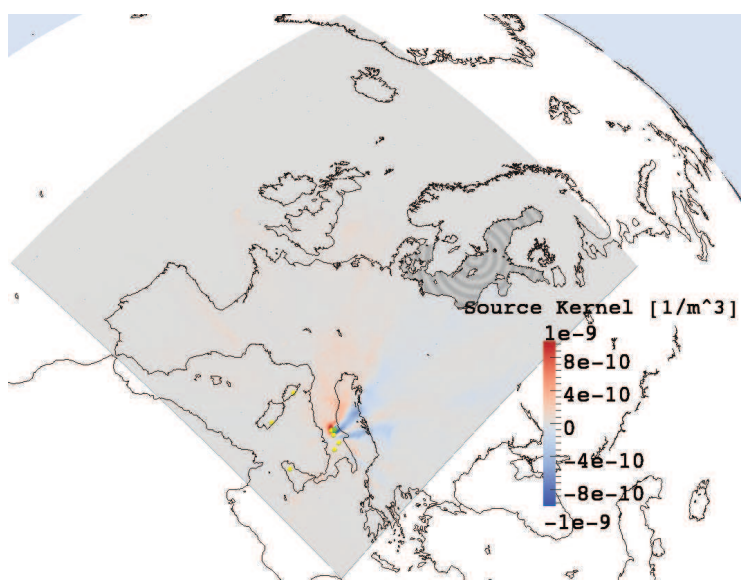

b)

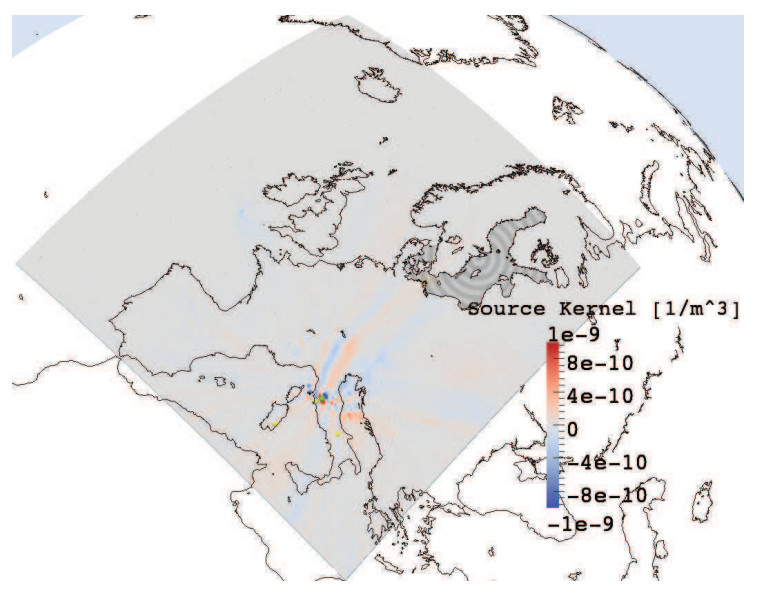

d)



Figure 8. Source sensitivity kernels, $K_{\sigma}$, for noise sources from the Baltic Sea, denoted by grey circles. (a) $K_{\sigma}$ for reference seismic station ARSA (green dot). It was possible to define an higher number of adjoint source, yellow dots, than in the case for reference stations ARCI and BSSO, respectively panels (b) and (c). The high sensitivity area in the eastern part of the region can not be simply associated with the geographical position of station ARSA with respect to the others, as we can see from figure 9 . (b) $K_{\sigma}$ for reference seismic station ARCI (green dot). (c) $K_{\sigma}$ for reference seismic station BSSO (green dot). (d) $K_{\sigma}$ kernel obtained as the sum over 26 reference-station kernels. There are three main lobes pointing towards three different region: the Mediterranean Sea, the Atlantic Ocean, and the eastern part of the study area. 


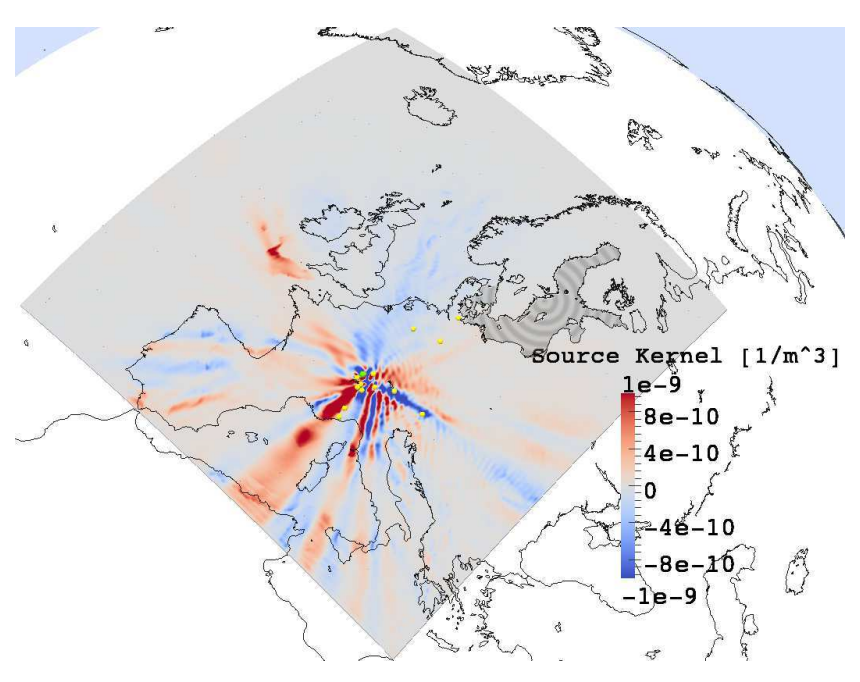

Figure 9. $K_{\sigma}$ for reference seismic station BRANT (green dot). Noise sources are located only in the Baltic Sea and are denoted by grey circles. Even if the geographical position of the reference station with respect to the others suggests the sensitivity to be concentrated in the western part of the study area, it is possible to see a strong "jet" of sensitivity protruding eastwards. 
a)



c)



b)

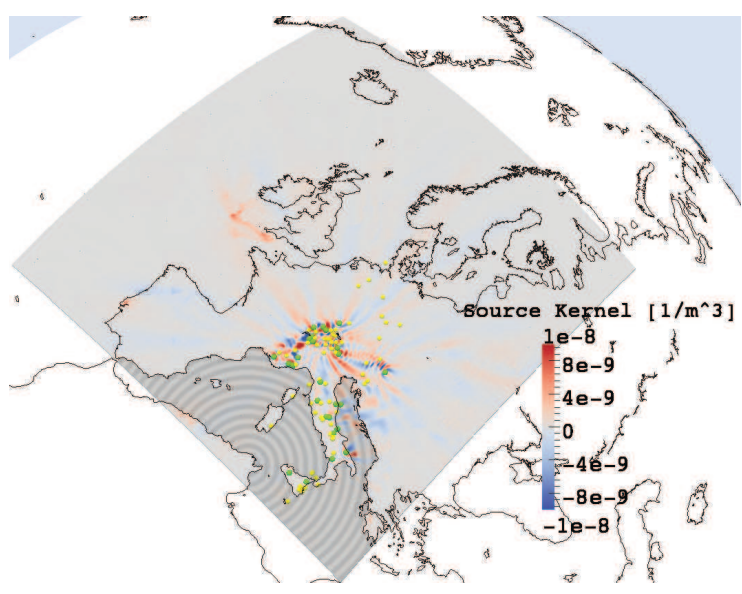

Figure 10. Source sensitivity kernels, $K_{\sigma}$, for noise sources from the Mediterranean Sea, represented by grey circles. (a) $K_{\sigma}$ computed for reference station ARSA (green dot). The stations used to construct the sensitivity kernel, yellow dots, are different from the ones used in figure 8: if we do not consider ambient noise coming from the Baltic region then the misfit between synthetic and observation-based correlograms exceed the limit we impose to build the adjoint sources. However the sensitivity is still concentrated in the eastern region. (b) Source kernel obtained as the sum of 26 single contributions and for a distribution of sources concentrated only in the Mediterranean Sea. The comparison with figure 8 panel (d) shows how sensitivity towards both the Atlantic and the Mediterranean decreases remarkably. However it is still possible to observe some sensitivity in the eastern region. (c) $K_{\sigma}$ computed for reference station BRANT (green dot). 
a)

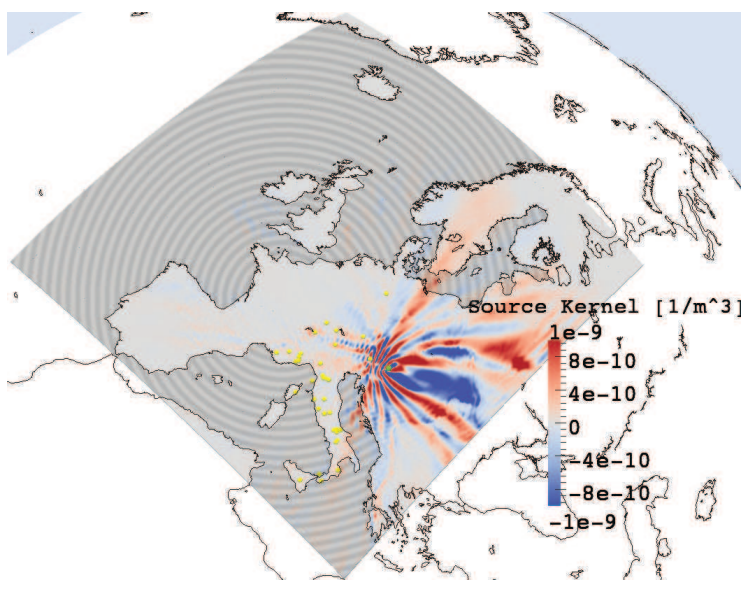

c)

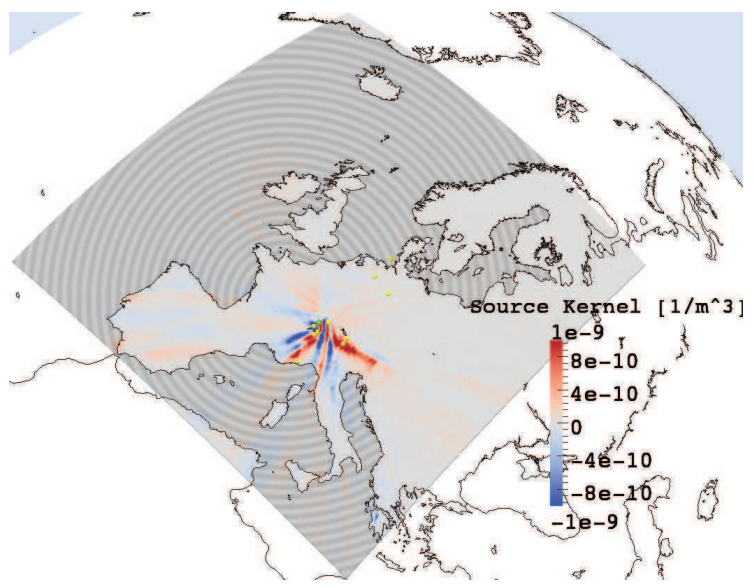

b)

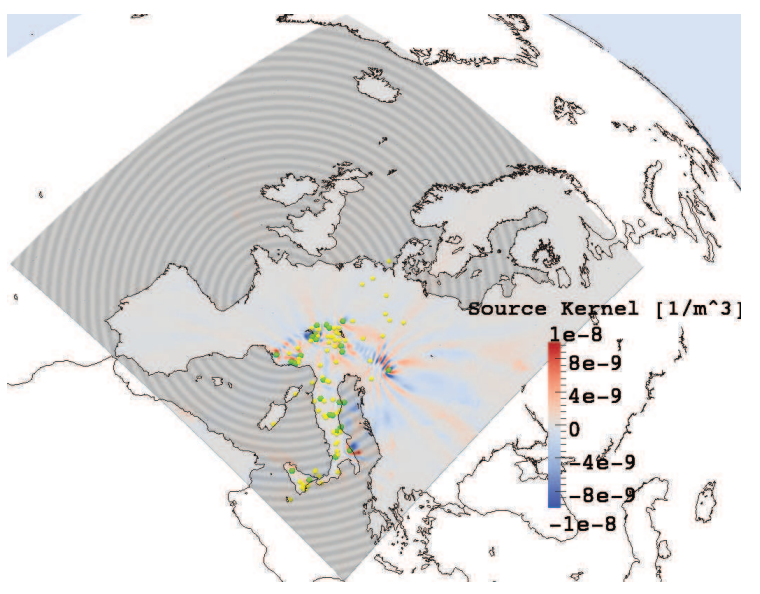

Figure 11. (a) $K_{\sigma}$ computed for reference station ARSA (green dot) and noise sources, denoted by grey circles, located in all the sea regions. For this figure are valid the same considerations we did in figure 8 panel (a). (b) $K_{\sigma}$ obtained as the sum of 26 single contributions and for a distribution of sources distributed throughout all sea regions. The sensitivity area near the Atlantic and the one in the Mediterranean are almost reduced to zero. It is still possible to observe lobes of sensitivity pointing towards east. (c) $K_{\sigma}$ computed for reference station BRANT (green dot) and noise sources located in all the sea regions. 
a)



c)

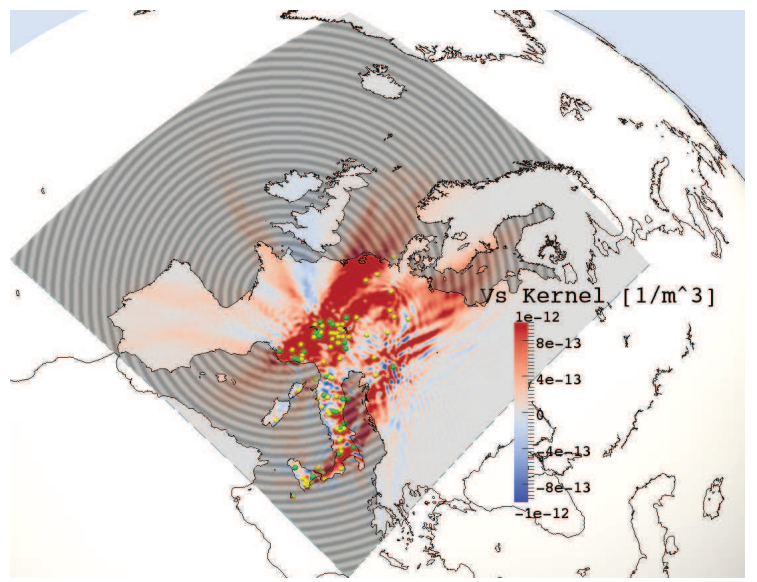

b)

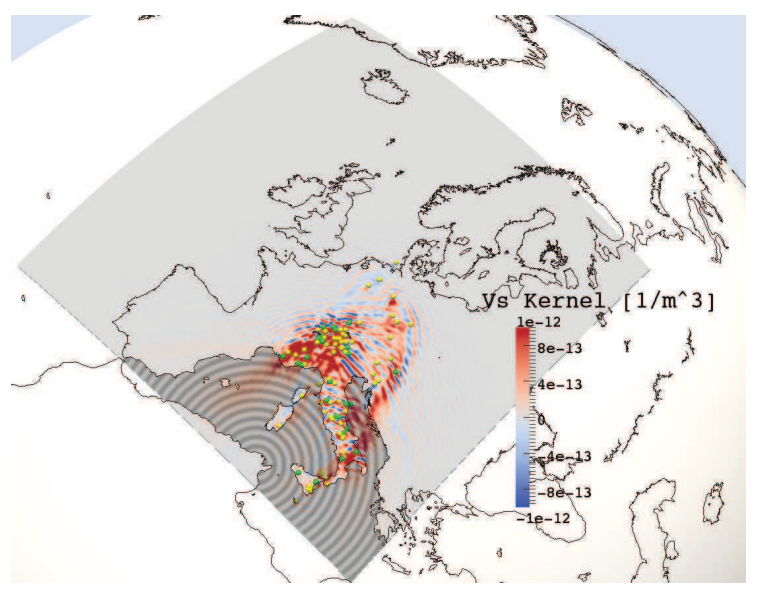

Figure 12. $\quad K_{\beta}$ kernel obtained as the sum of 26 different contributions for three different distributions of noise sources, represented by grey circles in the pictures: noise sources distributed in the Baltic Sea, panel (a), in the Mediterranean, panel (b), and in all the sea regions, panel (c). All the kernels are taken at a depth of $30 \mathrm{~km}$. Sensitivity is characterized by positive values in all three cases, indicating that our 3D shear background model is too slow. Areas illuminated by the sensitivity drastically change when we consider different distributions of noise sources: in panel (b) there is no sign of sensitivity in the area of the Baltic Sea, and both panel (a) and (b) do not show any sensitivity in France or Spain, as happens in panel (c) instead. 
a)

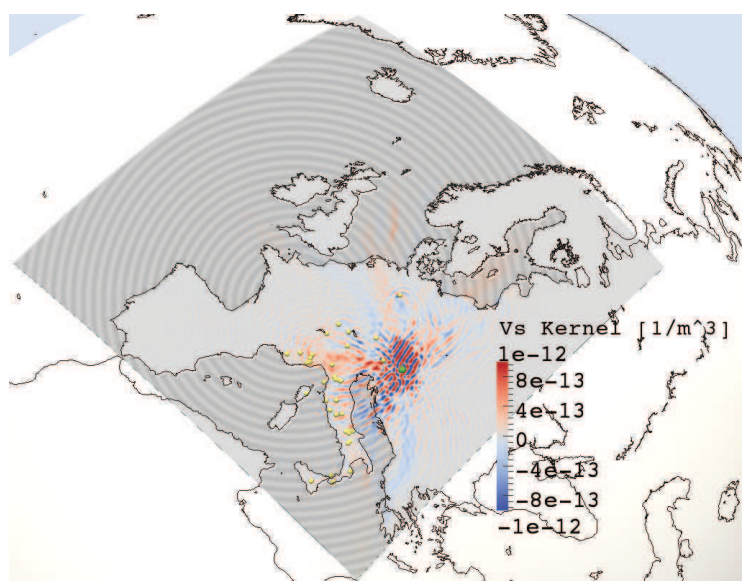

c)

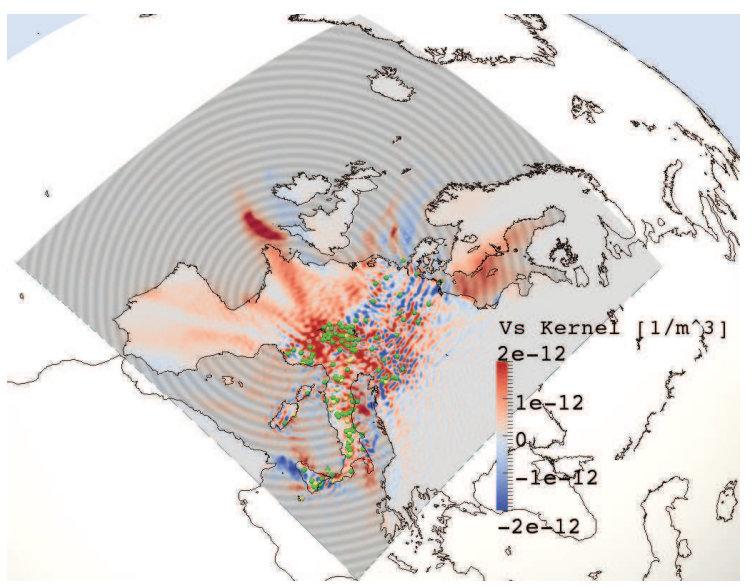

b)

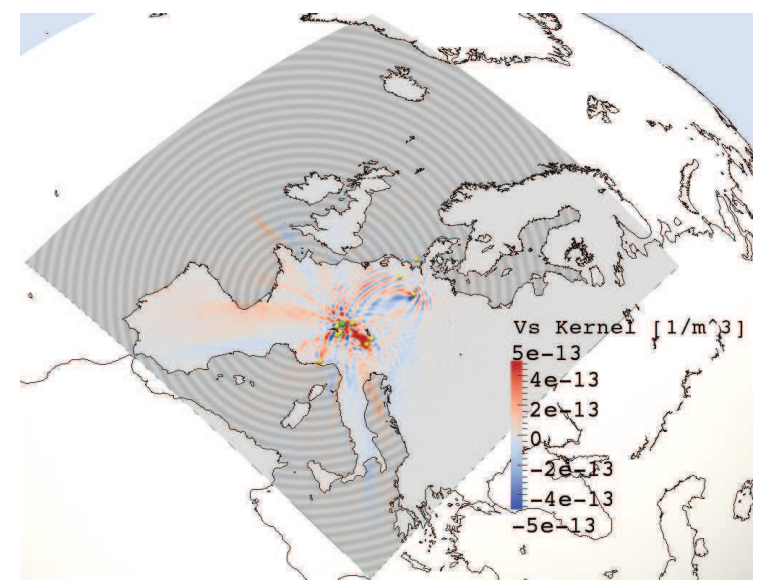

d)

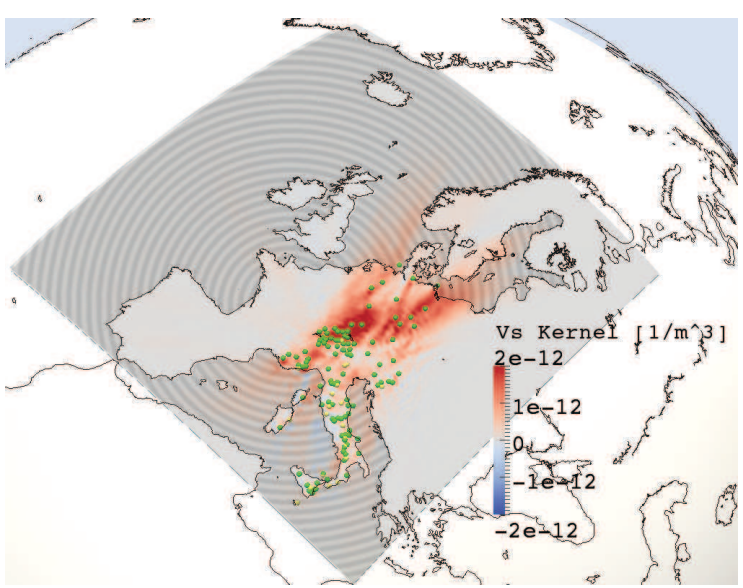

Figure 13. Structure sensitivity kernels, $K_{\beta}$, for noise sources located in all the sea regions, denoted by grey circles. Panels (a) and (b): slice of $K_{\beta}$ computed respectively for reference station ARSA and BRANT (green dots) taken at a depth of $10 \mathrm{~km}$. Panel (a) presents higher values of sensitivity with respect to panel (b) as happened in the case of $K_{\sigma}$, this will influence also the shape and values of the misfit kernel. Panels (c) and (d): slices of $K_{\beta}$ kernel taken respectively at 10 and $70 \mathrm{~km}$. The kernel is obtained as the sum of 104 different contributions. At $10 \mathrm{~km}$ depth Alpine region shows strong positive sensitivity, areas with negative values are concentrated mainly in the northeast part of the study region. At $70 \mathrm{~km}$ depth the sensitivity is almost completely positive indicating how the model we used has to be corrected with higher values of $\beta$. 
a)

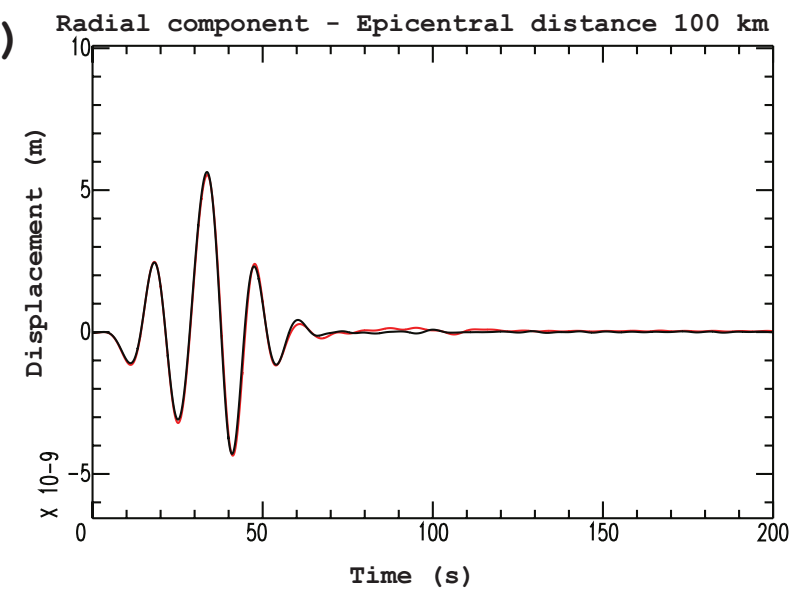

C)

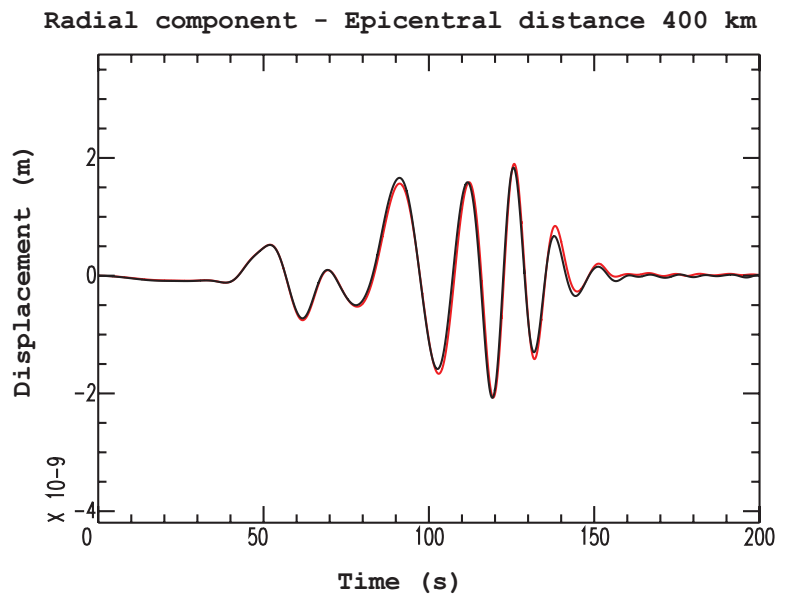

b)

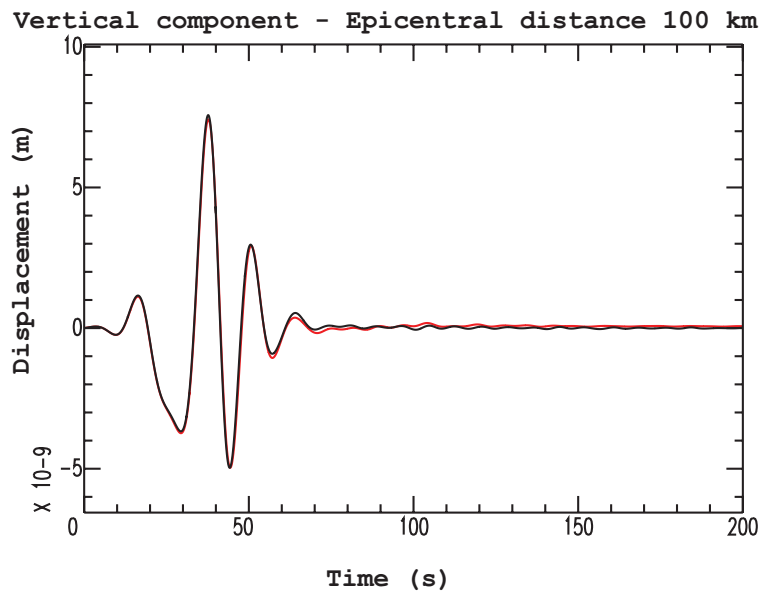

d)
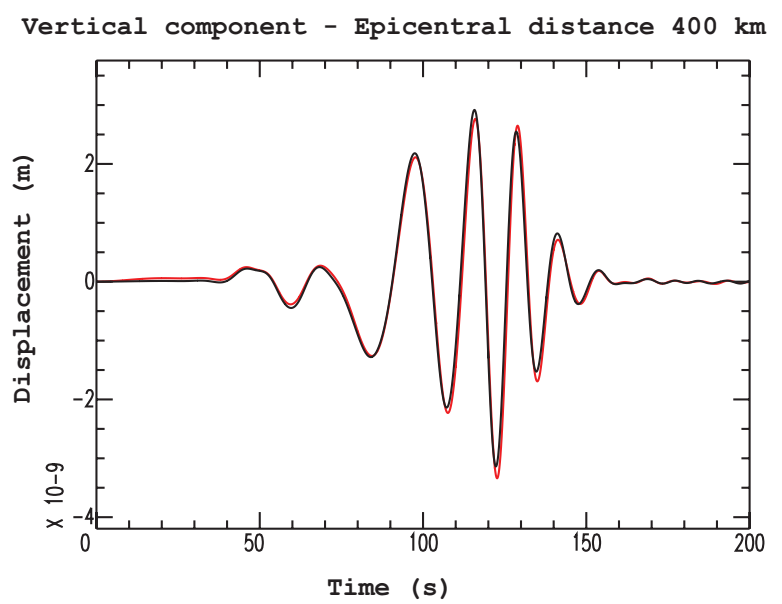

Figure 14. Results for the benchmark test between SPECFEM3D version 2.0, red lines, and SPECFEM3D_GLOBE, black lines. We placed a cross-shaped array of seismic receivers with interstation distance of about $100 \mathrm{~km}$ and we simulated a $10 \mathrm{~km}$ deep explosion at the center of the array. We applied a bandpass Butterworth filter between 10 and $30 \mathrm{~s}$. We have omitted the transverse components which are zero. 